\title{
Gust loading factors for tension leg platforms
}

\author{
Kurtis Gurley \& Ahsan Kareem \\ Department of Civil Engineering, University of Notre Dame, Notre Dame, Indiana 46556, USA
}

(Received 25 August 1992; accepted 8 February 1993)

\begin{abstract}
Utilization of tension leg platforms for deep water oil recovery has increased the importance of accurately predicting the effects of wind on these structures. The gust loading factor approach incorporates the effect of deviations from mean wind speed, gustiness, on structures based on extreme value statistics. Single-point representation of the wind field may be employed for structures smaller than typical gust size, but partial wind velocity correlation over large structures necessitates the use of multi-point wind field statistics to determine dynamic load effects. The characteristics of the wind field over the ocean are reviewed, and a previously reported description of an ocean-based wind spectrum is further examined in the light of additional full-scale offshore measurements. A modified coherence function for offshore applications is presented based on theoretical considerations and experimental data. A random vibration-based formulation of gust loading factors is presented that accounts for the hydrodynamic damping imparted by the platform motions in waves and currents. The response statistics of an offshore platform are predicted in the light of parametric uncertainties by a Monte Carlo simulation using the gust loading factor approach. This is used as the basis for a reliability analysis of the platform.
\end{abstract}

\section{INTRODUCTION}

The increasing demand on the performance of wind sensitive structures has placed a growing importance on the problem of wind effects on constructed facilities. As depth increases, the construction and maintenance of conventional jacket type oil platforms becomes less cost efficient. The tension leg platform (TLP) is a promising concept for deep water drilling. A TLP is a buoyant platform that is vertically moored to the seabed by pretensioned tethers. It is compliant in the horizontal plane and its motions have time periods that fall in the energy spectrum corresponding to the wind excitation frequency range. The importance of dynamic wind effects is therefore more significant on compliant structures than on conventional offshore platforms, where structural frequencies exceed the range of the wind energy spectrum.

A number of studies concerning the dynamic effects of wind on tension leg platforms have been reported in the literature. A sampling of these studies are found in Refs $1-7$.

Wind gustiness or turbulent fluctuation from mean velocity must be accounted for in the design of structures. For the case of large offshore structures, the stochastic modeling of the wind field over the ocean must include a multi-point representation to account for the partial spatial correlation of wind velocity over the structure. This correlation is expressed through the use of a coherence function whose form differs depending on the characteristics of the wind field under consideration. Aerodynamic admittance functions based on quasisteady and strip theories are then used to relate the wind field to the wind force over the structure. The coherence of wind fluctuation is an important flow characteristic in determining the aerodynamic admittance function. The rotational response in the horizontal plane (yaw) is particularly sensitive to the level of the coherence.

A gust factor based on extreme value excursion statistics represents the most probable extreme velocity value and is used for determining equivalent static loading. ${ }^{8}$ The gust factor approach can be extended to response statistics to express the most likely extreme response value, which again relies on the model representing coherence function. The concept of gust loading factors is based on statistical theory of buffeting and was first derived for land-based structures by Davenport. ${ }^{8}$ This concept for land-based structures has been modified by a number of investigators and it has gained worldwide acceptance in codes and standards for estimating alongwind load effects on structures (e.g. Simiu \& Scanlan). ${ }^{9}$ 
The uncertainties associated with various parameters related to the wind load effects introduce variability in the dynamic response estimates. These uncertainties in parameters arise from variability in the wind environmen, meteorological data, wind structure interactions, and structural properties. The complexity of the dynamic wind load effects, compounded by lack of a complete understanding of all the mechanisms that relate them to the far-field turbulence, and scarcity of both full-scale and experimental data have introduced significant levels of variability in their estimates. The concept of uncertainty in stochastic modeling of the load environment must be extended to uncertainties in structural properties in order to realistically assess the reliability of acceptable structural performance. A parameter study of various system properties can determine which properties the structure is most sensitive to, thus isolating problem areas where deck motion or fatigue may be especially important to performance.

This paper includes the theoretical background of wind field representation and correlation over large structural systems, the development of the gust factor with example applications to a TLP study, and a brief discussion of the application of the present work to reliability analysis.

\section{THEORETICAL BACKGROUND}

\section{Wind field representation}

The wind field in the horizontal lane is described in terms of a mean and a fluctuating velocity component as

$$
u(y, z, t)=\bar{U}(z)+\tilde{u}(y, z, t)
$$

where $u=$ the total instantaneous velocity, $\bar{U}=$ the mean velocity and $\tilde{u}=$ the fluctuating velocity, $y, z, t$ are horizontal and vertial translation, and time, respectively. The mean velocity is a function of height above the surface and is represented by either a logarithmic or power law. The logarithmic law is expressed as

$$
\bar{U}(z)=u_{s} 2 \cdot 5 \ln \frac{z}{z_{o}}
$$

Here $u_{s}=$ the friction velocity, which is a measure of turbulence over terrain of given roughness. $z_{o}=$ the surface roughness length, which can be related to the surface drag coefficient $C_{z}$ by the relationship ${ }^{6}$

$$
z_{o}=z \mathrm{e}^{-0.4} / \sqrt{C_{z}}
$$

Experimentally determined expressions for the surface drag coefficient $C_{z}$ have been developed, an example reported by Large \& Pond ${ }^{10}$ for $z=10$ metres is given as

$$
C_{10}=10^{-3}\left(0.49+0.065 \bar{U}_{10}\right)
$$

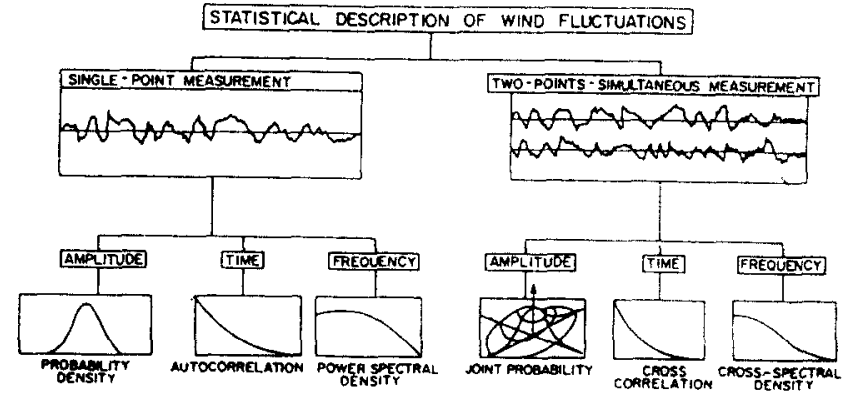

Fig. 1. Statistical description of wind fluctuations.

The power law, expressed as

$$
\bar{U}(z)=\bar{U}_{r}\left(\frac{z}{z_{\mathrm{r}}}\right)^{\alpha}
$$

is frequently used to describe mean velocity as a function of height. Here $\bar{U}_{\mathrm{r}}=$ the mean reference velocity, $z_{\mathrm{r}}=$ the reference height, $\bar{U}(z)=$ the velocity at height $z$, and $\alpha=$ an exponent that varies with terrain or sea state, and typically varies between $0 \cdot 1$ and $0 \cdot 15$ for wind over the ocean. ${ }^{9}$ The fluctuating wind component is statistically described as the second moment or root mean squared (rms) velocity. The variation in $\mathrm{rms}$ velocity with height is expressed in terms of turbulence intensity by

$$
I_{u}(z)=\frac{\sigma_{u}(z)}{\bar{U}(z)}
$$

where $\sigma_{u}=$ the rms of velocity $u$. The ESDU model for turbulence intensity is given by ${ }^{11}$

$$
\frac{\sigma_{u}(z)}{u_{s}}=\frac{7.5 \eta\left[0.538+0.09 \ln \left(z / z_{o}\right)\right]^{p}}{1+0.156 \ln \left(u_{s} / f_{\mathrm{c}} z_{o}\right)}
$$

where

$$
\eta=1-6 f_{\mathrm{c}} z / u_{s} \quad \text { and }=\eta^{16}
$$

and $f_{\mathrm{c}}$ is a Coriolis parameter. Equation (7a) may be transformed to turbulence intensity in the form of eqn. (6) through the use of eqn. (2) as

$$
I_{u}(z)=\frac{\sigma_{u}(z)}{\bar{U}(z)}=\frac{\sigma_{u}(z)}{u_{s}} \frac{u_{s}}{\bar{U}(z)}
$$

Other expressions for turbulence are available for various terrain conditions.

\section{Single-point wind field}

The wind velocity field over a structure may be described by single or multi-point statistics (Fig. 1). The statistics of a single point describe the wind behavior at a point in space, and are used to determine a number of properties. Turbulence intensity is a measure of the magnitude of wind velocity fluctuation. The autocorrelation function is a measure of the wind field's memory or current value dependence on the velocity value at a previous time. From this the power 


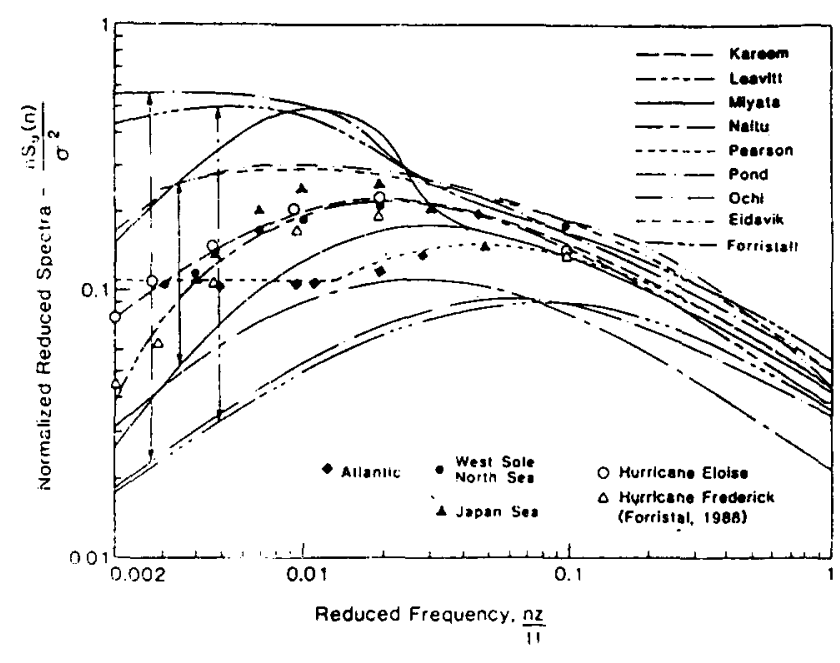

Fig. 2. Ocean-based wind spectra.

spectral density and length scale are determined. The power spectral denisty (PSD) describes the energy content in a fluctuating process as a function of frequency. The length scale is a measure of the size of turbulent eddies which determines the level of spatial correlation of wind gusts across the structure.

There are many models available to represent the PSD of fluctuating wind velocity. All the models exhibit similar trends in the high requency range, but show wide scatter at low frequencies. This scatter is largely due to the difficulty in confirming model characteristics in this frequency range by experiment. The statistical requirement to achieve the resolution necessary for low frequency data reduction is a very long sampling time. However, when the sampling time becomes too large the stationarity of the signal becomes questionable. Designers of land based structures have little interest in the low frequency range due to the relatively high natural frequencies of land systems, but the low frequency range becomes very important in the design of ocean-based compliant systems where resonance at very low frequencies is possible. The surface over which the wind field is being modeled also affects the wind spectrum. The standard models include parameters to account for varying terrain conditions on land but can not adequately represent the various forms of surface roughness due to wind-wave interaction over the ocean. Large waves move almost as fast as strong winds and contribute little to the surface roughness, while smaller waves may more significantly affect the wind velocity profile due to their tendency to break. A model for the wind spectrum over the ocean has been proposed by a number of researchers. ${ }^{4,5,12,13}$ An ocean-based spectral model derived by Kareem is given by

$$
\frac{f G_{u}(f)}{u_{s}^{2}}=\frac{C n}{(1+B n)^{5 / 3}}
$$

Here $G_{u}(f)=$ the single sided spectral density, $f=$ the frequency in Hertz, $n=$ the reduced frequency defined as $n=f z / \bar{U}$, and $C$ and $B$ are determined from environmental parameters. For $\bar{U}(10)=20 \mathrm{~m} / \mathrm{s}$ the values for $C$ and $B$ are computed to be 335 and 71 , respectively. Analysis of ocean wind data has shown that the proposed model adequately describes the fullscale data from the Gulf of Mexico, North Sea, Pacific and Atlantic oceans. Additional improvements are being studied to incorporate the effect of air-sea temperature difference and nonlinear interactions between different frequency components near the sea surface where turbulence is a maximum. ${ }^{14}$ Figure 2 compares several ocean-based wind spectra including the proposed spectrum and full-scale data. The model in eqn (8) provides a good match with the data and measurementsbased models.

\section{Multi-point wind field}

Single point representation tacitly assumes a fully correlated field, i.e. every point on the structure experiences the same wind fluctuations at the same time. This is valid for small, point-like structures such as small billboards, street lights and small floating ocean structures. For large structures the assumption of full correlation is unrealistic and it is necessary to relate the velocity fluctuations at one location to another through partial correlation. This multi-point wind field representation may be used to determine fluctuating wind loads at different locations on the structure.

The degree of correlation may be represented in either the time or frequency domain. The time domain representation expresses how well the velocity at the two locations are correlated for a range of time lags. In the frequency domain the cross power spectral density between velocities at two different locations is a measure of the degree of correlation. This description is expressed in terms of the coherence function between the two locations as

$$
G_{u_{i} u_{j}}(f)=\sqrt{S_{u_{i}}(f) S_{u_{j}}(f)} \operatorname{coh}(\Delta y, \Delta z, f) \mathrm{e}^{\mathrm{i} 2 \pi f \varphi}
$$

Where $G_{u_{i} u_{i}}(f)=$ the cross power spectral density between locations $i$ and $j$, and $\varphi$ is the phase. A commonly used expression for the coherence function is

$$
\operatorname{coh}(\Delta y, \Delta z, f)=\exp \left\{\frac{-f\left[\left(C_{v} \Delta z\right)^{2}+\left(C_{h} \Delta y\right)^{2}\right]^{1 / 2}}{\bar{U}_{12}}\right\}
$$

where $C_{h}$ and $C_{v}$ are experimentally determined decay constants which usually vary between 10 and $16, \Delta y$ and $\Delta z$ are the separation distance in the $y$ and $z$ directions, and $\bar{U}_{12}$ is the average velocity between the two locations. ${ }^{8}$ For the case of compliant offshore structures, the wavelength-to-reference-height ratio is large, indicating that the sea surface will influence the wind 


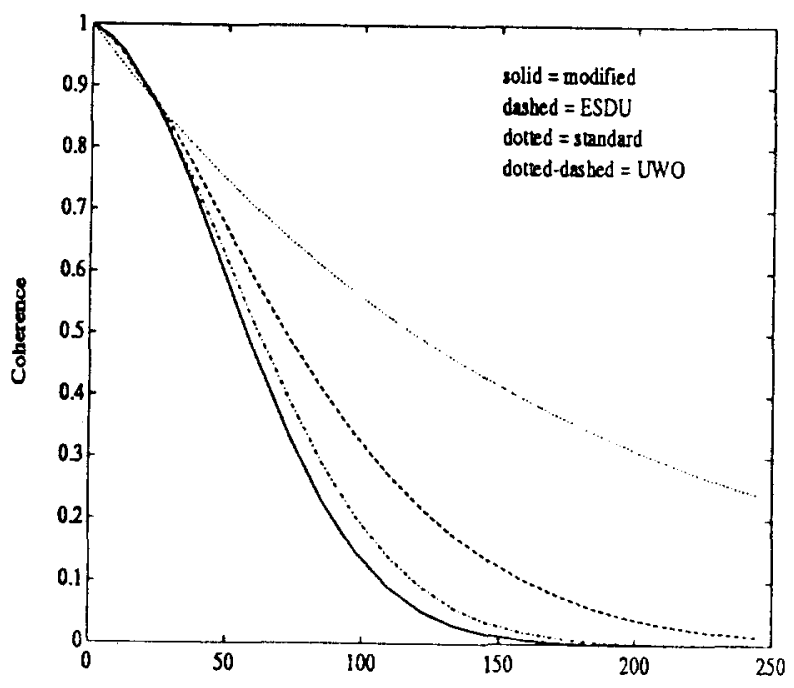

Fig. 3. Coherence versus horizontal separation (m).

field. When the separation between points is not small compared to the length scale of turbulence, it is important that the coherence expression contains the length scale parameter. An expression for the coherence of ocean-based structures that includes the length scale term has been proposed ${ }^{15}$ as

$$
\operatorname{coh}(\Delta y, \Delta z, f)=\operatorname{coh}(\Delta y, f) \operatorname{coh}(\Delta z, f)
$$

where

$$
\begin{aligned}
& \operatorname{coh}(\Delta z, f)= \\
& \exp \left(\left\{-\left(a_{z} \frac{\Delta z}{l}\right)^{2}+\left(\frac{f}{\bar{U}_{12}}\right)^{2}\left(b_{z} \Delta z+\frac{C_{z}(\Delta z)^{2}}{Z_{12}}\right)^{2}\right\}^{1 / 2}\right)
\end{aligned}
$$

For $\operatorname{coh}(\Delta y, f)$, replace $\Delta z$ with $\Delta y$ and $a_{z}, b_{z}, c_{z}$ (determined to be $0 \cdot 5,2,10$ respectively) with $a_{y}, b_{y}, c_{y}$ $(1,2 \cdot 5,16$ respectively) in the above formulation for $\operatorname{coh}(\Delta z, f) . l=$ the length scale of turbulence, $\bar{Z}_{12}=$ the average height of the locations. Similar expressions have been proposed by others. ${ }^{16,17}$ Figures 3-5 compare three commonly-used coherence functions along with the proposed modified coherence function as a function of vertical and horizontal separation at a fixed frequency and as a function of frequency with separation held constant, respectively. The coherence functions in the figures are the standard (eqn (10)), modified (eqn (11), $\mathrm{UWO}^{6,17}$ and ESDU, ${ }^{18}$ formulations.

The form of coherence function used is important for TLP response prediction that is consistently conservative in all six degrees of freedom. Tests have shown that the standard coherence function given in eqn (10) tends to lead to a conservative estimation of loading in the alongwind direction due to an overestimation of the degree of correlation. This results in a conservative surge response estimate but an unconservative estimate for rotational response about the vertical axis (yaw). These torsional loads are a result of unbalanced loading about

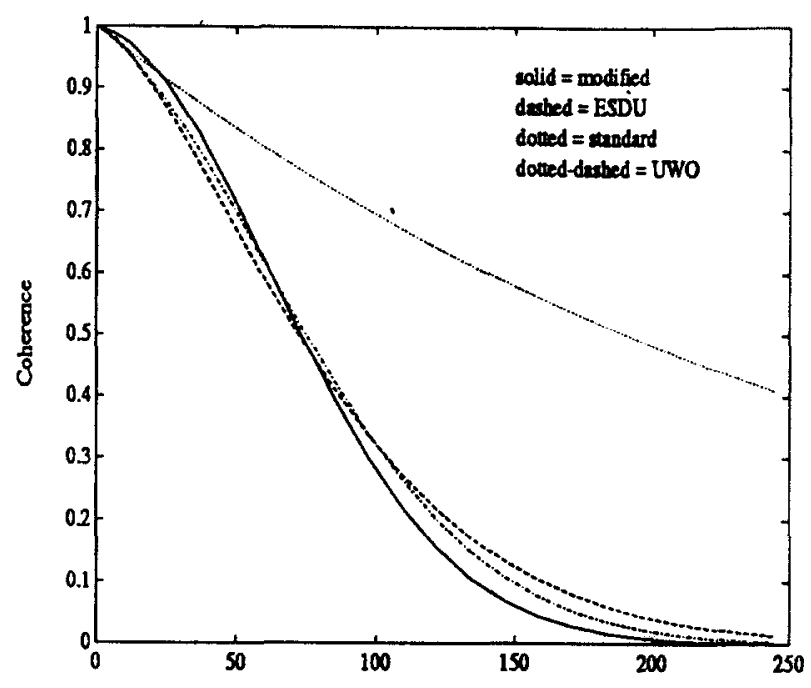

Fig. 4. Coherence versus vertical separation (m).

the vertical axis. Overestimates of correlation over the structure lead to less imbalance in the loading and thus an unconservative yaw response. The more conservative the correlation estimate, the less torsional loading results. It is therefore necessary to utilize the most accurate estimate of coherence available, since the use of a conservative coherence function will not necessarily yield conservative response predictions in all degrees of freedom of interest. Later in this paper an example is presented where the performance of the modified and standard coherence functions are compared for a TLP.

It is noted that for the design process, where conservative response estimates are the rule, it may be more practical to adopt a different worst case model for each type of response, rather than choosing one optimal model.

\section{Aerodynamic loading}

Once the multi-point wind field has been modeled, the

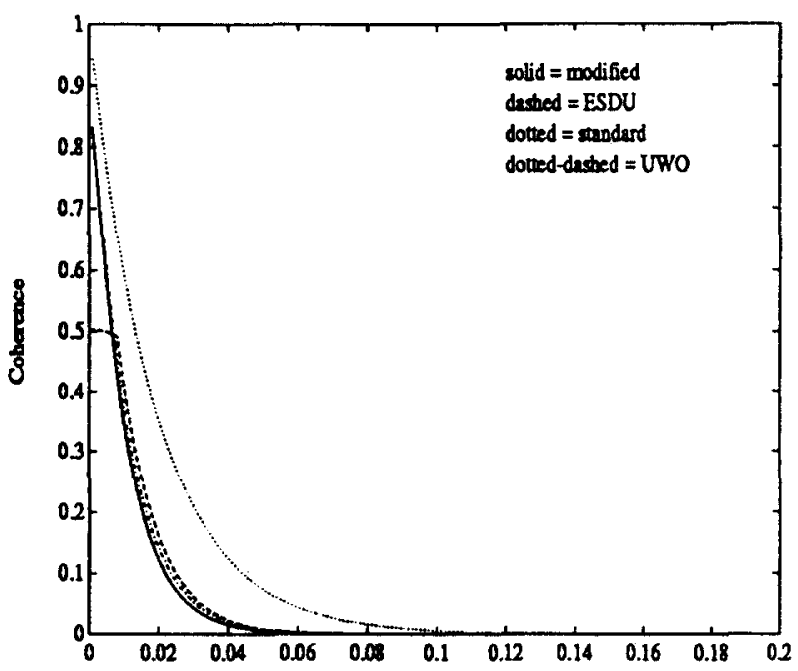

Fig. 5. Coherence versus frequency $(\mathrm{Hz})$. 
velocity field is transformed to the aerodynamic loading on the structure. The wind-induced loads may be represented by the superposition of steady and unsteady load components. The fluctuating wind velocity produces unsteady pressures on the surface of the structure that are a function of both time and position. The instantaneous pressure at a point may then be decomposed into a mean and fluctuating pressure. The loading for the simple case of a fully correlated structure may be expressed as

$$
F(t)=1 / 2 \rho A C_{\mathrm{D}}(\bar{U}+\tilde{u}(t)-\dot{x}(t))^{2}
$$

where $\rho=$ the air density, $A=$ the area of the structure, $C_{\mathrm{D}}=$ the drag coefficient, $\bar{U}$ and $\tilde{u}(t)$ are the mean and fluctuating wind velocity, and $\dot{x}(t)=$ the response velocity. The response velocity term is often ignored for stiff structures such as conventional jacket type platforms, but should be included for compliant structures, where large structural displacements may significantly affect the wind-wave loading functions.

The total force on a large structure may be evaluated by dividing the structure into smaller components over which full correlation is assumed and summing the force contribution from each component. The velocity field is simulated at the centroid of each component with each record matching the required power spectral density and also satisfying the desired level of correlation with respect to their spatial separation as specified by the coherence function.

In the time domain the response is evaluated by stepby-step integration of the equations of motion. This requires a time history description of the structural loading incremented in small enough steps to include any significant high frequency load effects. Autoregressive and moving average modeling (ARMA) is a technique available for time history simulation based on a desired spectral description. A multi-variate ARMA model may be used to generate a series of time histories with a desired level of correlation. ${ }^{19-22}$ Methods have been developed to increase the efficiency of ARMA modeling including tailoring the time increment to that required by the step-by-step integration scheme. ${ }^{19}$

The resulting total aerodynamic force is determined by

$$
F_{\mathrm{A}}(t)=\sum_{i=1}^{N} C_{i}^{\prime}\left[\bar{U}_{i}+\tilde{u}_{i}(t)-\dot{x}(t)\right]^{2}
$$

where $C_{i}^{\prime}=1 / 2 \rho C_{i} A_{i}, A_{i}=$ the area of $i$ th segment, $C_{i}=$ the aerodynamic force coefficient, $\rho=$ the air density, and $N=$ the total number of components. Assuming $\sigma_{x}<\sigma_{u}$ leads to the expansion of eqn (13) in terms of mean wind force, linear wind-exciting force, aerodynamic damping force, and quadratic wind force, respectively, as

$$
F_{\mathrm{A}}(t)=\bar{F}_{\mathrm{A}}+\tilde{F}_{\mathrm{A}}^{[1]}(t)-C_{\mathrm{A}} \dot{x}+\tilde{F}_{\mathrm{A}}^{[2]}(t)
$$

$$
\begin{aligned}
& \bar{F}_{\mathrm{A}}=\sum_{i=1}^{N} C_{i}^{\prime}\left(\bar{U}_{i}^{2}+\sigma_{u_{i}}^{2}\right) \\
& \tilde{F}_{\mathrm{A}}^{[1]}(t)=2 \sum_{i=1}^{N} C_{i}^{\prime} \bar{U}_{i} \tilde{u}_{i}(t) \\
& C_{\mathrm{A}}=2 \sum_{i=1}^{N} C_{i}^{\prime} \bar{U}_{i} \\
& \tilde{F}_{\mathrm{A}}^{[2]}(t)=\sum_{i=1}^{N} C_{i}^{\prime}\left[\tilde{u}_{i}^{2}(t)-\sigma_{u_{i}}^{2}\right]
\end{aligned}
$$

Here the nonlinear velocity terms are evaluated in terms of their mean square values at the $i$ th component denoted as $\sigma_{u_{i}}$.

In the frequency domain the input and output spectra of a system are related through transfer functions, and the total force spectrum is obtained through a transformation of the velocity spectrum. For point-like structures the force spectrum may be expressed as

$$
S_{F}(f)=\frac{4 F_{\mathrm{D}}^{2} S_{u}(f)}{\bar{U}^{2}}
$$

where $F_{\mathrm{D}}=1 / 2 \rho C_{\mathrm{D}} A \bar{U}^{2}$ and $S_{u}(f)$ is the spectral description of the wind velocity fluctuation.

For structures that are not fully correlated, the concept of multi-point statistics is used to include partial correlation effects. The linear wind force corresponding to eq (16) is expressed in terms of the cross-spectral density matrix of the wind field as

$$
G_{F_{\mathrm{A}}}^{[1]}(f)=4 \sum_{i=1}^{N} \sum_{j=1}^{N} C_{i}^{\prime} C_{j}^{\prime} \bar{U}_{i} \bar{U}_{j} G_{u_{i} u_{j}}(f)
$$

The cross-spectral density function of the quadratic wind force corresponding to eqn (18) is obtained by applying the frequency convolution approach, ${ }^{23}$ giving

$$
\begin{aligned}
G_{F_{\mathrm{A}}}^{[2]}(f)= & 4 \sum_{i=1}^{N} \sum_{j=1}^{N} C_{i}^{\prime} C_{j}^{\prime} \\
& \times\left[G_{u_{i} u_{i}}(f)^{*} G_{u_{j} u_{j}}(f)+G_{u_{i} u_{j}}(f)^{*} G_{u_{j} u_{i}}\right]
\end{aligned}
$$

where $G_{u_{i} u_{j}}$ is the single-sided cross-spectral density function of the velocity at locations $i$ and $j$, defined in eqn (9) and * represents convolution.

The difference between the time and frequency domain transformations of wind velocities to global forces is that the time domain transformation includes nonlinear feedback, where the frequency domain consists of linear and quadratic transformations, and linear feedback, shown in Figs $6 a$ and $6 b^{23}$

Previous work has shown that the rms contribution of the quadratic wind force component is small for typical ocean environment turbulence intensity values and may be ignored. ${ }^{23}$ The ratio of the variance of the linear and 

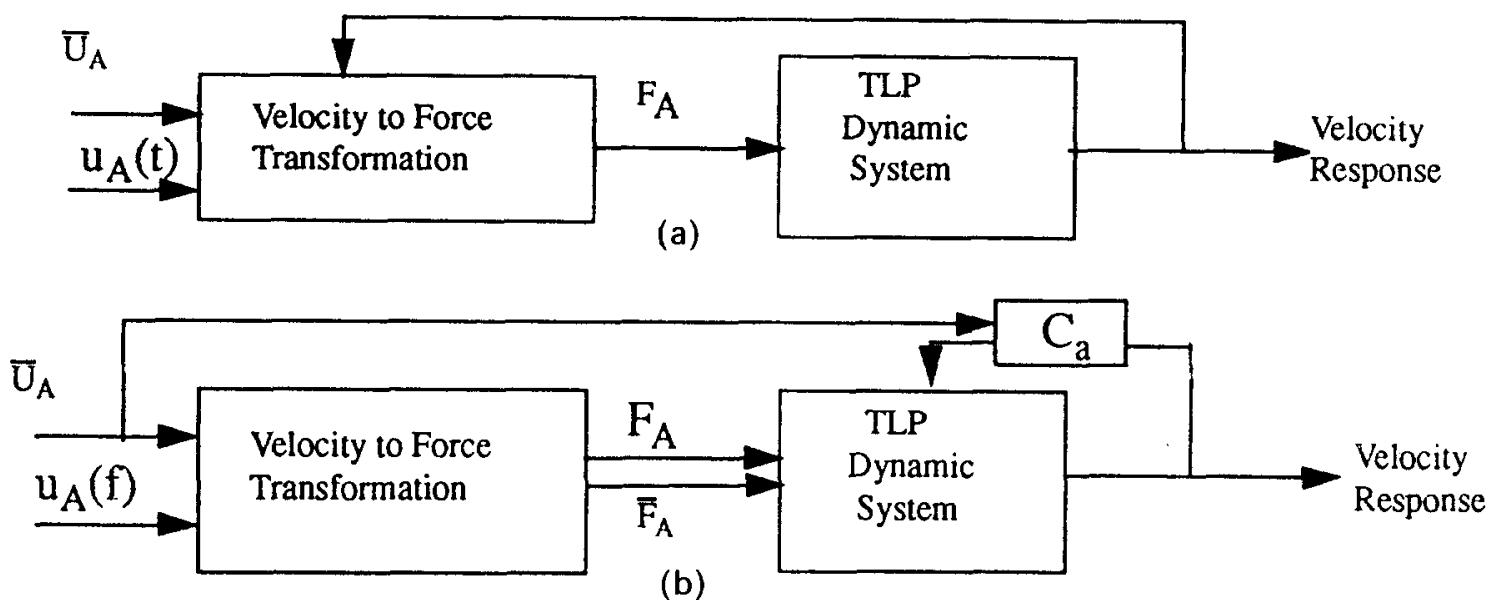

Fig. 6a. Time domain transformation. b. Frequency domain transformation.

quadratic terms in the expansion of wind forces, by virtue of the Gaussian nature of the wind fluctuations, can be expressed by

$$
\frac{\sigma^{[2]}}{\sigma^{[1]}}=\frac{I}{\sqrt{2}}
$$

Typical turbulence intensity is in the range of $15 \%$, so the quadratic force is generally about $10 \%$ of the linear force. These results are in agreement with the findings reported by Kareem. ${ }^{24}$ Examples using the Kareem, Davenport and Harris wind spectra ${ }^{24,25,26}$ are presented in Table 1. The example is that of wind acting on a TLP with an exposed area of $3376 \mathrm{~m}^{2}$, a drag coefficient of $1 \cdot 2$, and an air density of $1 \cdot 0$.

With the above simplification and assumption that the spectral description of the wind does not vary over the structure as a function of position i.e. $S_{u}\left(y_{i}, z_{i}, f\right)=S_{u}(f)$, eqn (19) may be rewritten as

$$
S_{F}(f)=\left(\rho A C_{\mathrm{D}} U\right)^{2} S_{u}(f) J_{F}(f)
$$

In this representation the partial correlation over the structure is accounted for by the aerodynamic admittance function $J_{F}(f)$, which may be derived on the basis of rapid distortion theory of turbulence or estimates using models of turbulent flow past bluff bodies. A standard admittance function for alongwind wind force is calculated based on quasi-steady and strip theories by integration of the standard coherence function (eqn. (10)) over the area of a rectangular plate. The result is

$$
J_{F}(f)=\frac{4}{\epsilon y^{2} \epsilon z^{2}}\left(\mathrm{e}^{-\epsilon y}+\epsilon y-1\right)\left(\mathrm{e}^{-\epsilon z}+\epsilon z-1\right)
$$

where $\epsilon y=f \theta C_{h} W / \bar{U}, \epsilon z=f \theta C_{v} D / \bar{U}$. $\theta=\left(\left(1+r^{2}\right) /(1+r)\right)^{1 / 2}, r=C_{h} W / C_{v} D, W$ and $D$ are the height and width of the rectangle. This closed form expression of $J_{F}(f)$ is applicable to structures easily modeled as lumped mass systems with simple geometry. Eqn. 24 is substituted into eqn. 23 to account for partial wind correlation of over large structures. Closed form expressions for pitch and yaw admittance functions correspondind to eqn 24 are derived by inclusion of a moment arm from each infinitesimal area to the axis of rotation in the integration and are expressed as

$$
\begin{aligned}
J_{M}(f)= & \frac{2}{3 \epsilon y^{2} \epsilon z^{4}}\left(\epsilon y+\mathrm{e}^{-\epsilon y}-1\right) \\
& \times\left(2 \epsilon z^{3}-6 \mathrm{e}^{-\epsilon z}-6 \epsilon z \mathrm{e}^{-\epsilon z}-3 \epsilon z^{2}+6\right)
\end{aligned}
$$

and

$$
\begin{aligned}
J_{r}(f)= & \frac{1}{3 \epsilon y^{4} \epsilon z^{2}}\left(\epsilon z+\mathrm{e}^{-\epsilon z}-1\right) \\
& \times\left(\epsilon y^{3}-12\left(\mathrm{e}^{-\epsilon y}+\epsilon y \mathrm{e}^{-\epsilon y}-1\right)\right. \\
& \left.-3 y \epsilon^{2}\left(\mathrm{e}^{-\epsilon y}+1\right)\right)
\end{aligned}
$$

For structures with complex shapes such as a TLP the rectangular idealization used in the closed form expression is inappropriate. The integration can be performed numerically by breaking up the structure into components and summing the appropriate coherence function in a double summation as

$$
J_{F}(f)=\sum_{i=j}^{N a} \sum_{j=1}^{N a} C_{\mathrm{D}_{i}} C_{\mathrm{D}_{j}} A_{i} A_{j} \bar{U}_{i} \bar{U}_{j} \operatorname{coh}(i, j, f)
$$

Here $\operatorname{coh}(i, j, f)$ is the same coherence originally defined

Table 1. Mean, linear and quadratic wind force components on a TLP in surge

\begin{tabular}{lccccc}
\hline Spectrum & $\begin{array}{c}\text { Mean wind } \\
\text { velocity } \\
(\mathrm{m} / \mathrm{s})\end{array}$ & $\begin{array}{c}\sigma \text { of } \\
\text { fluctuation } \\
(\mathrm{m} / \mathrm{s})\end{array}$ & $\begin{array}{c}\text { mean wind } \\
\text { force } \\
(\mathrm{kN})\end{array}$ & $\begin{array}{c}\sigma^{[1]} \\
\text { linear force } \\
(\mathrm{kN})\end{array}$ & $\begin{array}{c}\sigma^{[2]} \\
\text { quadratic } \\
\text { force }(\mathrm{kN})\end{array}$ \\
\hline Harris & $29 \cdot 6$ & $3 \cdot 39$ & $1798 \cdot 0$ & $406 \cdot 51$ & $32 \cdot 92$ \\
Davenport & $29 \cdot 6$ & $3 \cdot 22$ & $1795 \cdot 8$ & $386 \cdot 1$ & $29 \cdot 70$ \\
Kareem & $29 \cdot 6$ & $3 \cdot 81$ & $1804 \cdot 2$ & $456 \cdot 9$ & $41 \cdot 58$ \\
\hline
\end{tabular}


in eqn (9) with $(i, j)$ replacing $\Delta y, \Delta z$ to represent the separation between components.

This multiple discrete-area approach tailors the admittance function to the shape of the specific structure, allows for the use of individual drag coefficients for different components, interference effects, and approaches closed form accuracy as the structure is broken into smaller components. For complex geometries the closed form solution may be unattainable.

The formulation of pitch/yaw loading is accomplished by including a moment arm from the load component centroid to the axis of rotation of the overall structure

$$
J_{M, T}(f)=\sum_{i=j}^{N a} \sum_{j=1}^{N a} C_{\mathrm{D}_{i}} C_{\mathrm{D}_{j}} A_{i} A_{j} \bar{U}_{i} \bar{U}_{j} l_{i} l_{j} \operatorname{coh}(i, j, f)
$$

where $l_{i}$ is the moment arm of the $i$ th component in the vertical or horizontal plane for $J_{M}(f)$ (pitch) and $J_{T}(f)$ (yaw), respectively. It is noted that the closed form formulations of the admittance function in eqns (24)(26) are dimensionless, while those of eqns 27 and 28 are not. When applying the later admittance functions eqn (23) must be altered to $S_{F}(f)=\rho^{2} S_{u}(f) J_{F}(f)$.

Corresponding to the mean and fluctuation component representation of the wind velocity and force, the structural response may also be expressed as the superposition of a mean (static) and rms (dynamic) value. The formulations for static and dynamic response are presented in the next section, along with the formulation of the gust factor approach and its application to system input and output.

\section{Averaging interval}

Wind speed, response or any other continuous signal is always observed through a low-pass filter. An example of a filter is the anemometer used in wind speed measurements, where some time-averaging of the incoming signal is performed due to limitations in the anemometer's frequency response, the response period is expressed as the averaging time $T_{\mathrm{a}}{ }^{27}$ The effect of this inherent filtering is to underestimate the power spectral density in the high frequency range. Figure 7 shows the effect of a low-pass filter on the time history, probability density function, and power spectrum of a process $X(t)$, the filtered process is represented in the figure as $Y(t)$.

Another form of spectral distortion is from the sampling time $T_{\mathrm{s}}$ which is the chosen length of each realization in the ensemble. The sampling time is inversely proportional to frequency resolution in the spectral representation. When longer sampling times are used, the resolution of the spectrum becomes finer and spectral peaks are less likely to be averaged out. To minimize the error due to resolution (bias error), it is desirable to make the resolution as fine as possible by

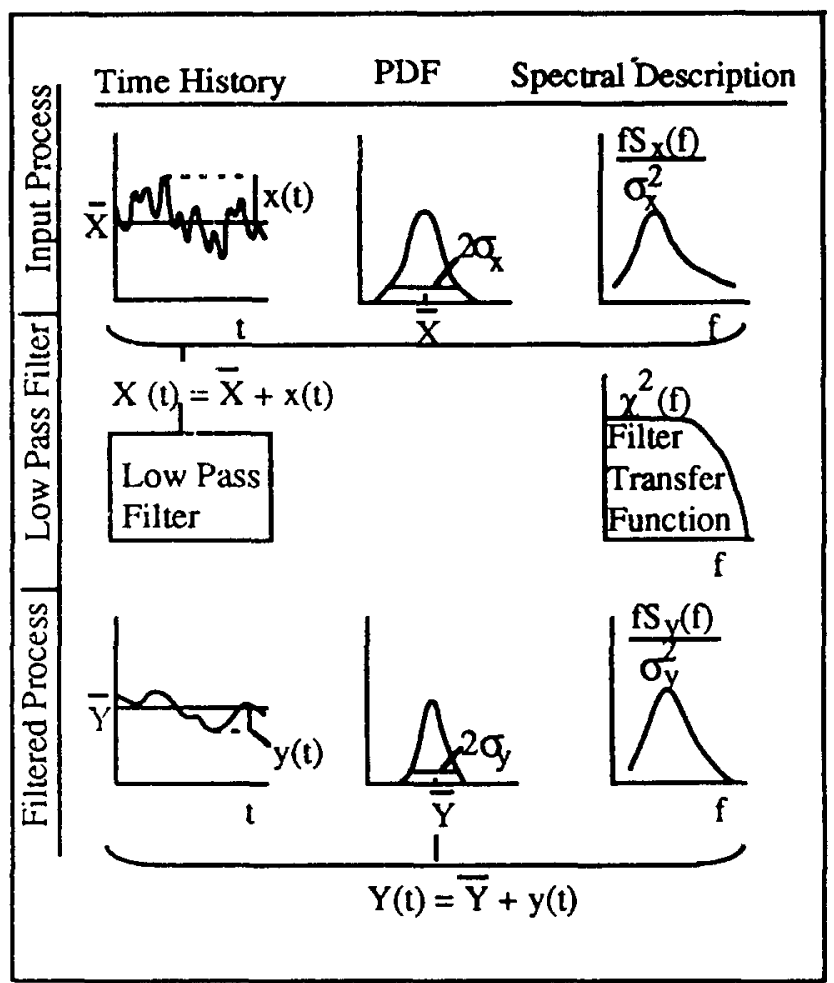

Fig. 7. Effect of low-pass filter on signal.

choosing a large sampling time. On the other hand, a longer sampling time means the number of realizations in the ensemble decreases. This increases the variance error, which is inversely proportional to the square root of the number of realizations. As discussed previously, a large sampling time also decreases the stationarity of the signal. A compromise between bias error and variance error must be reached based on experience and parameteric studies.

One of the advantages of using filters to account for the averaging and sampling periods is in the comparison of predicted response estimates based on model spectra to full-scale measurements. The predicted response is based on the actual averaging and sampling periods utilized during full-scale measurement and thus it provides a more consistent basis for comparison of predicted and measured response. The sampling and averaging time introduces a spectral window that truncates and attenuates the signal at low and high frequencies depending on the $T_{\mathrm{s}}$ and $T_{\mathrm{a}}$. In order to compare gust factors based on model spectral discriptions to those based on measured signals, these filters are either multiplied through the model spectra or divided out of the measured spectra. The filters are expressed as

$$
\chi_{\mathrm{a}}(f)=\left(\frac{\sin \left(\pi f T_{\mathrm{a}}\right)}{\pi f T_{\mathrm{a}}}\right)^{2}
$$

and

$$
\chi_{\mathrm{s}}(f)=1-\left(\frac{\sin \left(\pi f T_{\mathrm{s}}\right.}{\pi f T_{\mathrm{s}}}\right)^{2}
$$




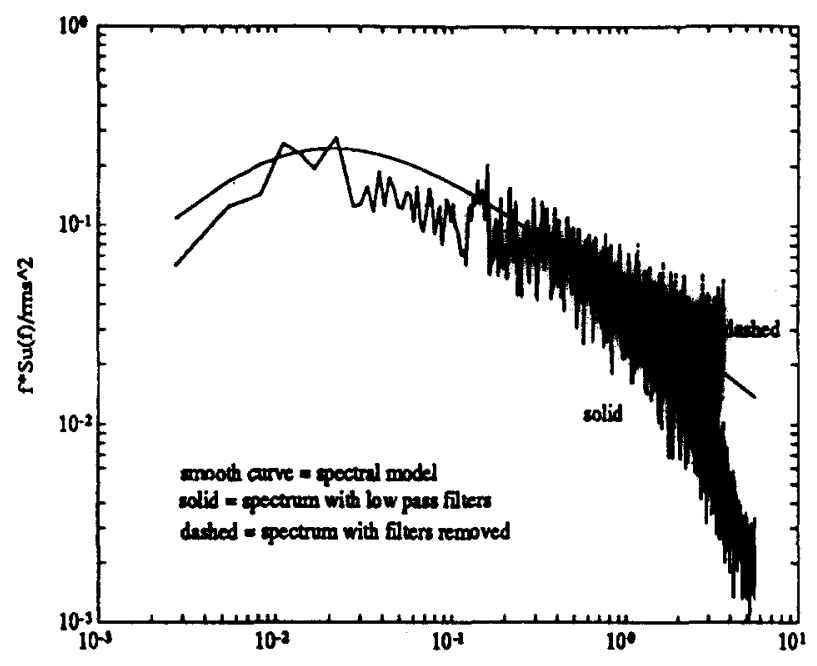

Fig. 8. Wind spectra with and without filters.

and applied to a spectral model by

$$
S_{u}\left(T_{\mathrm{a}}, T_{\mathrm{s}}, f\right)=S_{u}(f) \chi_{\mathrm{a}}(f) \chi_{\mathrm{s}}(f)
$$

where $S_{u}\left(T_{\mathrm{a}}, T_{\mathrm{s}}, f\right)=$ the wind spectrum as a function of sampling time, averaging time, and frequency. Figure 8 demonstrates the effect of these filters on the spectrum of a measured wind velocity signal. Figures 9 and 10 show the effects of averaging time and sampling time on the gust factor for several spectral wind velocity models. Figure 9 holds $T_{\mathrm{s}}$ at 600 seconds constant and varies $T_{\mathrm{a}}$ and Fig. 10 holds $T_{\mathrm{s}}$ at 3600 seconds and varies $T_{\mathrm{a}}$. Design specifications recommend different averaging times depending on the size of the structure, e.g. three seconds for cladding, roofing, and glazing, five seconds for elements with its largest vertical or horizontal dimensions less than $50 \mathrm{~m}$, and 15 seconds for structures with very large dimensions.

\section{GUST FACTOR}

A gust represents a large deviation in the mean wind.

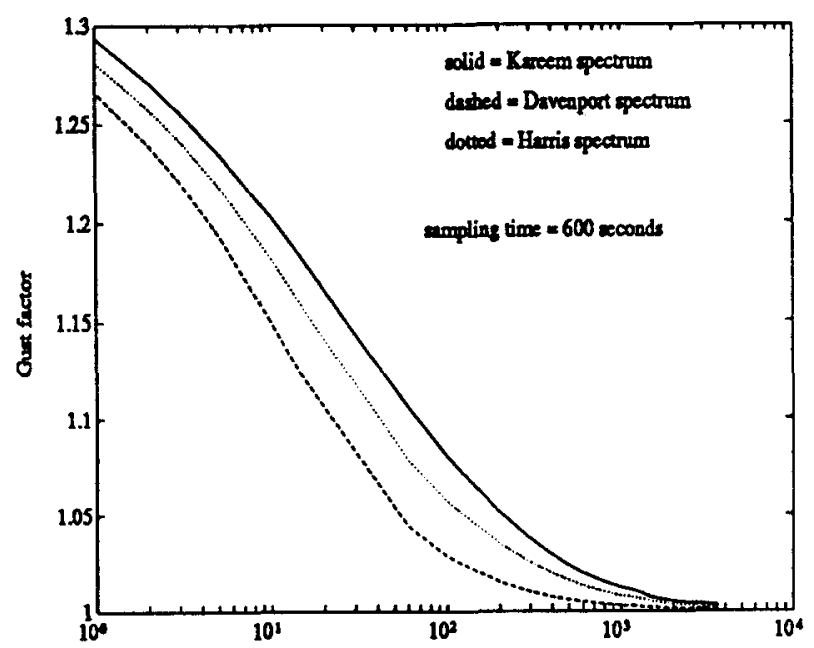

Fig. 9. Gust versus averaging time (s).

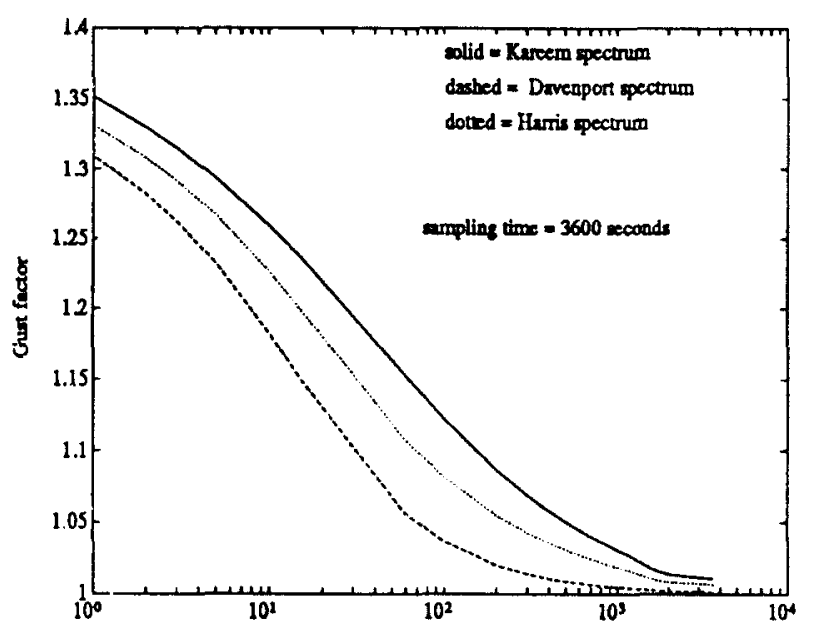

Fig. 10. Gust versus averaging time (s).

Wind gustiness introduces dynamic loading effects on the system, which can be examined in terms of a gust loading factor. In order to evaluate the peak response of the system, the peak wind load must be considered. Maximum load effects are due to correlated high pressure over the entire structure in the form of eddies at least the size of the structure. Eddies of small size compared to the structure impinge successively rather than instantaneously, hence small eddies are unable to correlate significant pressures over the whole structure. Eddies which are larger envelop the entire structure and hence are able to cause well correlated pressure.

Current design codes include the effect of gustiness by factoring up the maximum expected mean wind effects of given probability. For the simple case of point-like structures, the maximum loading due to wind gustiness is used as an equivalent static load to determine maximum displacement. The idea may be extended to large systems by evaluating the maximum gust loading on individual components and correlating their occurrence over the structure to find the most probable overall maximum loading.

The total instantaneous wind speed may be viewed as the sum of the mean and fluctuating components. The maximum wind speed during an interval $T$ may then be expressed as the summation of the mean value with the rms value multiplied by a peak factor $(g)$ statistically derived to express most probable maximum deviation from mean wind speed during interval $T$. It is written as

$$
U_{\max }=\vec{U}+g \sigma_{u}
$$

The peak factor $g$ is a statistical representation of the most likely extreme excursion in a signal, for a typical Gaussian process it is usually in the range 3-4. Various techniques have been developed to analyze a signal for extreme excursion statistics. The derivation of two common methods of excursion analysis, Rice (1944, $1945)^{28}$ and Gumbel $(1958)^{29}$ are presented in the Appendix.

Using one of the available methods of excursion 
analysis, a peak factor is calculated, and the gust factor is defined as

$$
G=\frac{u_{\max }}{\bar{U}}=1+\frac{g \sigma_{u}}{\bar{U}}
$$

From the discussion in the Appendix, the gust factor may be viewed as the deviation from the mean of the process $u(t)$ which, on average, is exceeded once during the reference period $T^{28}$

The gust factor may be calculated from measured data by dividing a record into a number of realizations, each equal in length to the desired sampling time $T_{\mathrm{s}}$. The sampling time should be large eoungh such that peak values from all realizations are statistically independent. The measured gust factor is then the average peak value from all realizations divided by the mean value of the total record. In this study, gust factors were computed for wind velocity data taken at sea at an elevation of 260 feet, and collected at a rate of $2 \mathrm{~Hz}$. An eleven-hour total record was used with realization lengths chosen from two to ten minutes. The gust was predicted using the spectrum given by eqn (8) in the form of eqn (31), and eqns (29), (30), (A5), (A8) and (33). The gust was measured for each hour and the full record and compared to the predicted values using an approximated averaging time of one minute. The maximum difference between measured and predicted gust factors for all cases was $3 \cdot 41 \%$, with an average difference of $1 \cdot 32 \%$.

\section{Gust loading factor}

A prediction of the maximum load effects or structural displacment, including the effects of amplification due to dynamic oscillation, is needed in the design process. The gust factor approach relies on gust factors that are based on random vibration theory to translate the dynamic amplification of loading caused by atmospheric turbulence and dynamic sensitivity of the structure, into an equivalent static loading. The gust factor can be used to estimate the expected maximum deviation from mean response. The response is represented be a mean (static) component and a fluctuating (dynamic) component. The formulation of static and dynamic response in the surge degree of freedom is presented here. The static response is simply the mean wind force divided by structural stiffness.

$$
\tilde{x}=\frac{F_{\mathrm{A}}}{k}
$$

where $F_{\mathrm{A}}=\frac{1}{2} \rho C_{\mathrm{D}} A U^{2}$, and $k=2 \pi f_{\mathrm{n}}^{2}$ where $f_{\mathrm{n}}$ is the system natural frequency in Hertz. The dynamic response is the square root of the area under the response spectrum, which is determined by multiplying the force spectrum by the system transfer function. The force spectrum is determined by a transformation of the wind velocity spectrum as given in eqn (23). The response variance is given by

$$
\begin{aligned}
\sigma_{x}^{2} & =\int_{0}^{\infty} S_{x}\left(T_{\mathrm{a}}, T_{\mathrm{s}}, f\right) \mathrm{d} f \\
& =\int_{0}^{\infty}|H(f)|^{2} S_{F}\left(T_{\mathrm{a}}, T_{\mathrm{s}}, f\right) \mathrm{d} f \\
& =\int_{0}^{\infty}|H(f)|^{2} S_{u}\left(T_{\mathrm{a}}, T_{\mathrm{s}}, f\right) J_{F}(f) \mathrm{d} f
\end{aligned}
$$

where $|H(f)|=$ the transformation function from loading to response and is determined by the governing equations of motion. A modified version of eqn (27) (with density squared multiplied in the formulation) is applied above as the admittance function. The integration of eqn (35) can be performed by separating the resonant and background response components. The residue theorem is used to evaluate the resonant response with the wind spectrum idealized as a white noise, and the response due to background effect is evaluated on the basis of a quasi-static assumption. Thus eqn (35) may be expressed as ${ }^{15}$

$$
\begin{aligned}
\sigma_{x}^{2}(r)= & \frac{\pi f_{\mathrm{n}} S_{F}(f)\left(2 \pi f_{\mathrm{n}}\right)^{2 r}}{4\left(2 \pi f_{\mathrm{n}}\right)^{4} \xi m^{2}} \\
& +\frac{\left(\int_{0}^{\infty} S_{x}\left(T_{\mathrm{a}}, T_{\mathrm{s}}, f\right) \mathrm{d} f\right)\left(2 \pi f_{\mathrm{n}}\right)^{2 r}}{\left(2 \pi f_{\mathrm{n}}\right)^{4}}
\end{aligned}
$$

where $r=0,1,2,3$ for displacement, velocity, acceleration, and jerk respectively, and $f_{\mathrm{n}}$ is the natural frequency of the system. The peak factor is now defined as in eqns (A5) and (A8) with the response spectrum $S_{x}$ applied in eqn (A5). The response gust factor is now

$$
G_{x}=1+\frac{g_{x}\left(\int_{0}^{\infty}|H(f)|^{2} S_{u}\left(T_{\mathrm{a}}, T_{\mathrm{s}}, f\right) J_{F}(f) \mathrm{d} f\right)^{1 / 2}}{\bar{x}}
$$

where $\bar{x}$ is the mean response, and $g_{x}$ is the peak factor of the response. Equations (35)-(37) may be used to predict pitch and yaw fluctuating responses by replacing the surge admitance function $J_{F}(f)$ by the applicable pitch or yaw admittance functions $J_{M}(f), J_{T}(f)$ respectively. In eqn (36) the force spectrum is replaced with a torque spectrum, mass is replaced by the inertia term and $S_{x}$ is replaced with $S_{\theta}$ for rotational displacement. The damping term $\xi$ in the first term on the right side of eqn (36) includes structural, aerodynamic and hydrodynamic components that will be explained in the following section.

As noted earlier, the contribition of the square of the fluctuating velocity component to the rms value of force is small, and is often ignored. However, a recent analysis of extremes based on the non-Gaussian distribution of the fluctuating loads that result from inclusion of 
nonlinear velocity terms exhibited slightly higher gust factor values. ${ }^{30}$ This increase in gust factor combined with the small increase in the rms value noted earlier may require closer examination of these effects in the determination of extreme loads.

\section{Damping}

For large ocean-based structures, the total damping in the system consists of the inherent damping of the material, and the damping due to the system motion in viscous fluids. These aerodynamic and hydrodynamic components vary in their contribution to the total damping for each degree of freedom, as the relative fluid-structure velocity differs in each degree of freedom. The aerodynamic and hydrodynamic effects contribute significantly to the surge response of compliant structures such as a TLP, due to the large displacements in this degree of freedom. All expression for the aerodynamic damping can be derived as

$$
\xi_{\mathrm{A}}=\frac{\rho_{\mathrm{air}} C_{\mathrm{D}_{\mathrm{a}}} A_{\mathrm{a}} \bar{U}}{4 \pi m f_{\mathrm{n}}}
$$

where $A_{\mathrm{a}}, C_{\mathrm{D}}, m$ indicate the area, mean drag coefficient above the mean water level, and total mass.

The overall hydrodynamic damping consists of radiation and drag-induced components that result from potential and viscous actions of waves on submerged portions of TLPs. The radiation damping contribution is relatively small, therefore, the draginduced component serves as the major source of damping available to dampen the TLP motions. The magnitude of the drag-induced hydrodynamic damping is dependent on the drag coefficient used in the Morrison's equation to express the hydrodynamic forces. The drag coefficient is dependent on the Keulegan-Carpenter (KC) number and the Reynolds number. For TLP applications, the drag coefficient needed corresponds to extremely low KC numbers and high Reynolds numbers for which experimental data are not available. Laboratory data for low KC numbers are available, but these are limited to relatively small Reynolds numbers. ${ }^{31-33}$

Recent advances in computational fluid dynamics have provided another avenue for the determination of the drag coefficient computationally. Both the experimental and numerical results suggest a sharp increase in $C_{\mathrm{D}}$ as the KC number approaches zero. ${ }^{34}$ This change points out that in this range of the $\mathrm{KC}$ numbers the drag mechanism experiences a change, i.e. the drag force which is primarily dominated by flow separation becomes less significant and Stokes-type drag becomes significant. ${ }^{35}$ Accordingly, a part of the drag force is represented by a linear function of the relative fluidstructure velocity and the others by a quadratic function generally used for separated flow. The turbulence in water through an increase in the effective shear further enhances the Stokes-type damping. An accurate quantification of the drag coefficient in these categories would require extensive experimental and computational data. In this manner the overall drag force can be expressed as a combination of the two components. Both numerical and experimental investigations are in progress at different research establishments to better understand the physics of viscous hydrodynamic damping to better model this important dissipation force that has very significant influence on the determination of gust loading factors as well as wave-induced forces.

The damping component linearly related to platform motion can be treated in a straightforward matter, whereas the quadratic component needs to be linearized to facilitate frequency domain analysis. The quadratic hydrodynamic contribution is obtained by an equivalent linearization approach and may be broken down into damping due to current and damping due to waves. Their total contribution may be expressed as

$$
\xi_{\mathrm{W}_{2}}=\frac{\rho_{\mathrm{w}} C_{\mathrm{D}_{\mathrm{w}}} A_{\mathrm{w}} \sigma_{u}}{2\left(2 \pi f_{\mathrm{n}}\right) m} \frac{1}{2}\left(\left(2 \sqrt{\frac{2}{\pi}}\right) \mathrm{e}^{-\ddot{U}_{r}^{2} / 2}+2 \bar{U}_{\mathrm{e}}^{2} \operatorname{erf}\left\langle\frac{\bar{U}_{\mathrm{c}}}{\sqrt{2}}\right\rangle\right)
$$

where $\rho_{\mathrm{w}}, C_{\mathrm{D}}, A_{\mathrm{w}}$ are the water density, coeficient of drag under water, and the area under the mean water level, $\bar{U}_{\mathrm{c}} \sigma_{u}$ are the mean current velocity and rms particle velocity in the waves. ${ }^{36,37}$ The total damping ratio can be represented by the summation of the structural, aerodynamic, and hydrodynamic damping ratio as

$$
\xi=\xi_{\mathrm{s}}+\xi_{\mathrm{A}}+\xi_{\mathrm{W}_{1}}+\xi_{\mathrm{W}_{2}}
$$

In which $\xi_{\mathrm{W}_{1}}$ represents linear drag damping expressed as

$$
\xi_{\mathrm{W}_{1}}=\frac{\gamma \rho \pi h \sqrt{v \sum D_{i}}}{m \sqrt{4 \pi \mathrm{f}_{\mathrm{n}}}}
$$

where $\rho=$ water density, $h=$ column draft, $v=$ kinematic viscosity, $\Sigma D_{i}$ is the summation of the TLP column and pontoon diameters, $f_{\mathrm{n}}=$ surge natural frequency, $m=$ mass, and $\gamma$ is unity for Stokes damping. An $\gamma$ higher than unity is expected due to turbulence in water.

The above approach is based on the assumption that wind and wave effects can be treated separately. However, their effects may be coupled, and due to nonlinearities this assumption may not always be valid. By considering an equivalent quadratization approach instead of equivalent linearization, additional damping contributions may be obtained. This is an area of current research in the field.

\section{UNCERTAINTY ANALYSIS OF GUST FACTORS}

In the analysis of large systems there are many 
assumptions and approximations that are made during the modeling of the loading environments and system response. Given the stochastic nature of both system input and output, the gust factor estimate will need to include a measure of uncertainty. The nature of modeling necessarily introduces these uncertainties into the analysis, since many system parameters and material properties experience spatial and temporal variations that render deterministic representation unrealistic. For a TLP system some examples include tether crosssectional area and tether yield strength, system mass, damping and natural frequency, as well as uncertainties inherent in the loading environment. These uncertainties may be propagated through a Monte Carlo simulation or similar techniques by defining the uncertain parameters in terms of their first and second moments and a probability distribution. During the simulation each parameter is assigned a random value based on its description. The resulting system gust response factor includes the effects of the uncertain parameters in terms of first and second moments.

A sensitivity analysis of the various parameters may be performed by modeling the quantities as deterministic and varying one parameter at a time incrementally through many simulations. This parameter study can determine which parameters the structure is most sensitive to and which should receive extra care in design, construction and quality control.

\section{Reliability analysis}

Uncertainty may be viewed as a measure of the risk of failure or serviceability of a system. The convention in the past was to design wind-excited structures for equivalent static loads, with uncertainties expressed in the form of safety factors. In order to properly assess the safety and reliability of a structure, uncertainties in the system may be used to calculate the associated probability of failure. The propability of failure more specifically is the probability of exceeding performance levels necessary for a particular role. Any complicated system has more than one mode of failure. In the case of a TLP, failure may be breakage of tendons, displacements that render the platform inoperational, or acceleration levels that exceed those needed for human performance.

Reliability analysis is accomplished by examination of the limit state function designed to determine conditions leading to a failure mode. The limit state equation is generally of the form

$$
F=R-S
$$

where failure is indicated by the condition $F<0$. The resistance $R$ and load $S$ are random variables which may be replaced by the expressions necessary in describing the particular mode of failure. Unlike customary strength analysis the limit state for human performance requirement may be described such that $R$ and $S$ would represent human acceleration tolerance and structural acceleration response, respectively. Another example is the limiting of the TLP maximum offset for proper operation of the production risers. The two basic variables in the limit state are usually functions of a number of other variables, each with its own statistical description. The analysis becomes complex if some or all of the variables are non-Gaussian or if the limit state involves nonlinear relations, as is the case for TLP loadstructure interaction. Closed form evaluation of the failure probability is almost always computationally prohibitive and in most cases requires information that is not available. An approximate solution using first and second moment statistics involving linearization of the limit state is known as the first-order reliability method (FORM). The limit state surface is expanded in therms of a Taylor series, and only first-order terms are retained. The limit state is then represented by a hyperplane at the point on the surface with the highest probability density. If terms up to second order are retained in the Taylor series expansion the method is referred to as second-order reliability method (SORM) where the limit state representation is a quadratic surface. An alternative procedure is the use of a Monte Carlo simulation which requires the probability distribution functions of the limit state variables. A simulation is then executed and the probability of being in the failure zone $(F<0)$ is determined.

For modes with a very low probability of failure, a straightforward Monte Carlo simulation requires significant computer time to achieve an accurate result. Various techniques are available to limit the sampling space to areas of interest; this is known as importance sampling. One method is the implementation of the gust factor approach to reliability analysis. Using the gust loading factor approach the load may be described by its extreme value distribution. This distribution may be used for the load term in the limit state equation, thus limiting the sampling space to the region of interest, i.e. the region where catastrophic failure is most likely to occur. The extreme loading function for TLP surge motion may be expressed by

$$
S=G \sum_{i=1}^{N a} \frac{1}{2} \rho C_{\mathrm{D}_{i}} A_{i} \bar{U}_{i}^{2}
$$

where $G$ here is the estimate of wind gustiness and $i$ is the platform component number being summed over the structure. For the case of pitch and yaw wind loading a moment arm term is included in eqn (42). From the probability density function of extreme loading the probability of exceeding typical design loading levels may be identified. This information is used to design a structure to resist wind loads for a target value of probability of exceedence (POE). For example, if the TLP is to be designed for loads with a $7 \%$ POE in the 


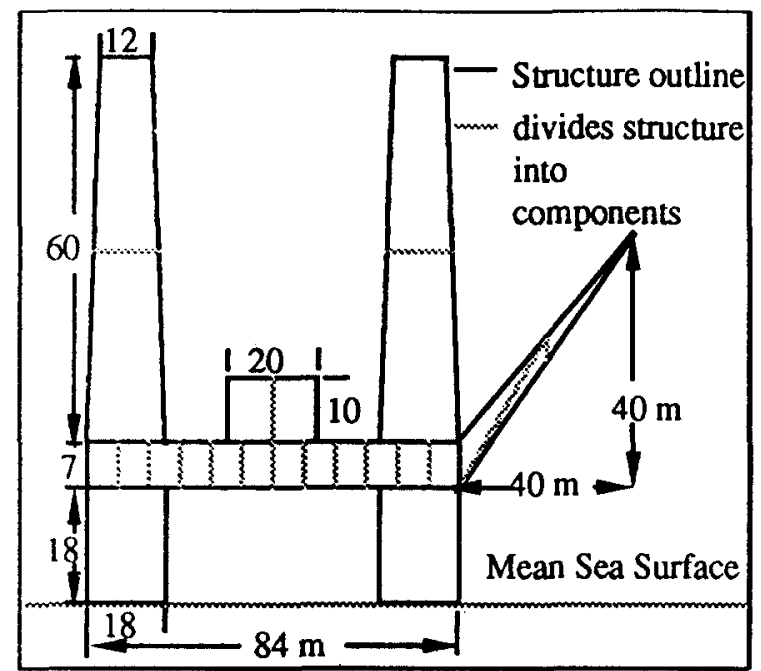

Fig. 11. Schematic of tension leg platform used in example.

twenty-year design life of a platform, the extreme loading distribution may be used to determine the loading value for which there exists a $7 \%$ chance of being exceeded in the reference time. This value provides a framework for load and resistance factor design for the TLP.

A simple reliability analysis is performed for the TLP under consideration in the next section using Probabilistic Analysis (PROBAN) software. ${ }^{38}$ FORM, SORM, Monte Carlo simulation, and Directional simulation (a variance reduction form of simulation) methods are compared to find the probability of exceeding a maximum allowed displacement in the surge direction. Statistics of the total response due to wind are calculated using a stochastic gust factor analysis (described in the next section), and used in the limit state function as the load variable. A maximum displacement tolerance is then defined and the probability of exceedence is simulated. Results are described in the following section.

For most systems, catastrophic failure is not likely due to a single passage of some critical loading value. More often, structural members fail due to fatigue caused by many cycles of moderate stress loading and unloading (moderate referring to the ratio of cyclic

Table 2. Deterministic parameters in TLP simulation

\begin{tabular}{ll}
\hline \multicolumn{1}{c}{ Parameter } & \multicolumn{1}{c}{ Value } \\
\hline Generalized mass & $7 \cdot 0 \mathrm{E} 7 \mathrm{~kg}$ \\
Generalized moment of intertia in pitch & $2 \cdot 6 \mathrm{E} 9 \mathrm{~kg} \mathrm{~m}^{2}$ \\
Generalized moment of inertia in yaw & $2 \cdot 6 \mathrm{E} 9 \mathrm{~kg} \mathrm{~m}$ \\
Structural Damping ratio & $0 \cdot 05$ \\
Area under water & $1340 \mathrm{~m}^{2}$ \\
Area under wind & $3376 \mathrm{~m}^{2}$ \\
Air mass density & $1 \cdot 0 \mathrm{~kg} / \mathrm{m}^{3}$ \\
Water mass density & $1000 \mathrm{~kg} / \mathrm{m}^{3}$ \\
Exponent of the velocity field & $0 \cdot 12$ \\
Current velocity & $1 \cdot 0 \mathrm{~m} / \mathrm{s}$ \\
Wave particle velocity & $2 \cdot 0 \mathrm{~m} / \mathrm{s}$ \\
Axial tendon stiffness & $5000 \mathrm{~N} / \mathrm{m}$ \\
\hline
\end{tabular}

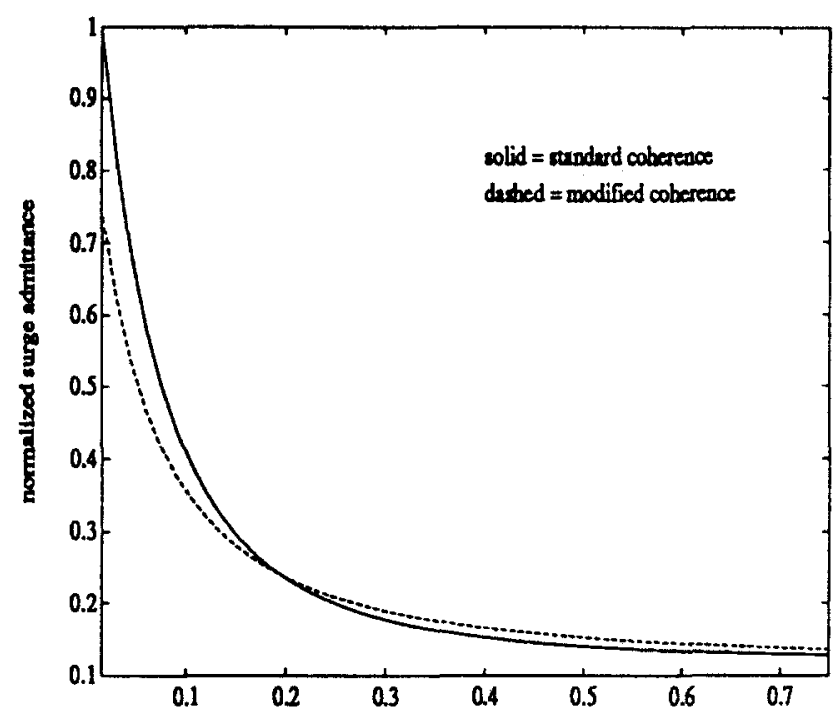

Fig. 12. Surge admittance versus frequency $(\mathrm{Hz})$.

stress to critical stress). In some cases it may be appropriate to define the limit state function to reflect the effects of fatigue including crack initiation and growth. This must be considered when using extreme value distributions for loading in the limit state. Fatigue reliability analysis may require a loading function representing daily events rather than just the extremes.

\section{TLP EXAMPLE}

The concept of gust factors and uncertainty analysis is applied to a TLP to predict its response statistics to wind loading over the ocean. Figure 11 is a view of the TLP being considered. Table 2 lists the values of the deterministic parameters used in the simulation and Table 3 lists the uncertain parameters and their statistics. The hydrodynamic damping related to quadratic damping is only considered here. The framework provided here can include contribution by the linear drag term once additional data becomes available. In

Table 3. Uncertain parameters in TLP simulation

\begin{tabular}{lcc}
\hline Random variable & Mean & COV \\
\hline$C$ (eqn (8)) & $335 \cdot 0$ & $0 \cdot 25$ \\
$B$ (eqn (8)) & $71 \cdot 0$ & $0 \cdot 19$ \\
Surge natural frequency & $0.010 \mathrm{~Hz}$ & 0.20 \\
Pitch natural frequency & $0.45 \mathrm{~Hz}$ & 0.20 \\
Yaw natural frequency & $0.015 \mathrm{~Hz}$ & 0.20 \\
Drag coefficient in air & 1.14 & $0 \cdot 20$ \\
Drag coefficient in water & 0.50 & 0.30 \\
Turbulence intensity & 0.10 & 0.20 \\
$C y$ (decay constant) & 16.0 & 0.25 \\
$C z$ (decay constant) & 10.0 & $0 \cdot 15$ \\
Extreme wind velocity & $45.17 \mathrm{~m} / \mathrm{s}$ & 0.097 \\
at centroid & & \\
Extreme value param. & Location & Scale \\
$\quad$ for wind & parameter & parameter \\
At a height of 10 meters & 27.931 & 2.89 \\
\hline
\end{tabular}




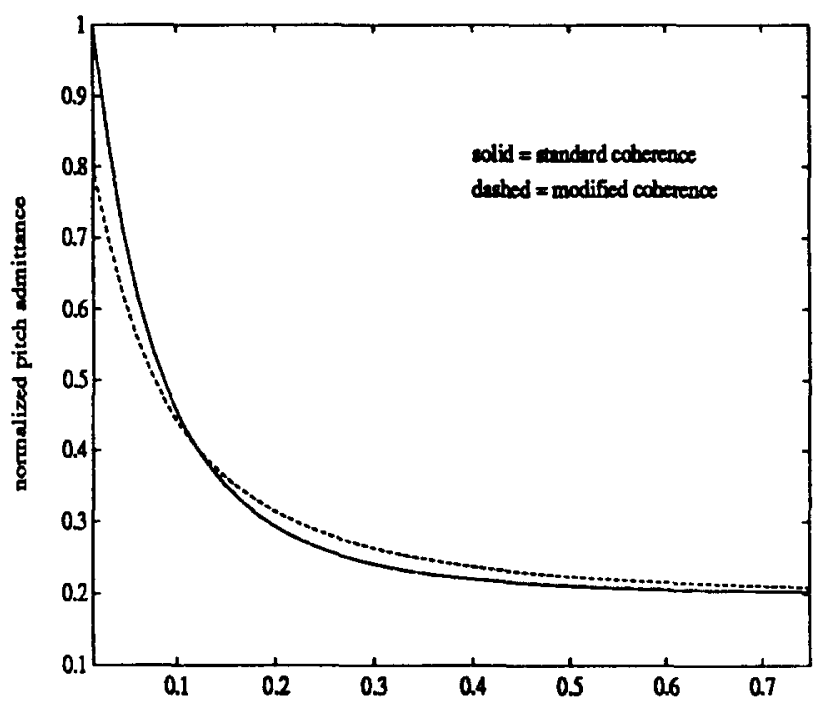

Fig. 13. Pitch admittance versus frequency $(\mathrm{Hz})$.

the light of this, additional uncertainty is assigned to the underwater drag coefficient. In Table 3 the first 10 uncertain variables are simulated with a Gaussian distribution while the extreme wind velocity is simulated with a Gumbel distribution.

The simulation consists of assigning random values to the various uncertain parameters described based on their assigned probability density function. The wind field is simulated using the ocean spectrum given in eqn (8) for the frequency domain-based fluctuating response calculation, and a 20 -year extreme value distribution based on full-scale ocean measurements is used to simulate the wind speed for the mean response calculation. Both the static and dynamic responses are represented in terms of mean and rms components. The gust response factor is calculated in each simulation from the static and dynamic response and the peak factor (eqns (34)-(37), (A5), (A8), and the first and

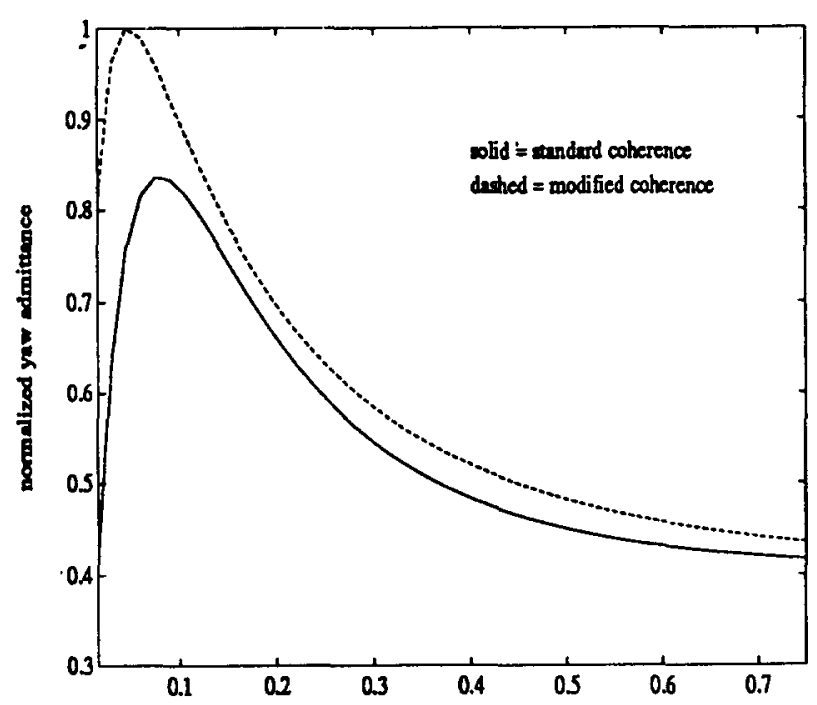

Fig. 14. Yaw admittance versus frequency $(\mathrm{Hz})$.

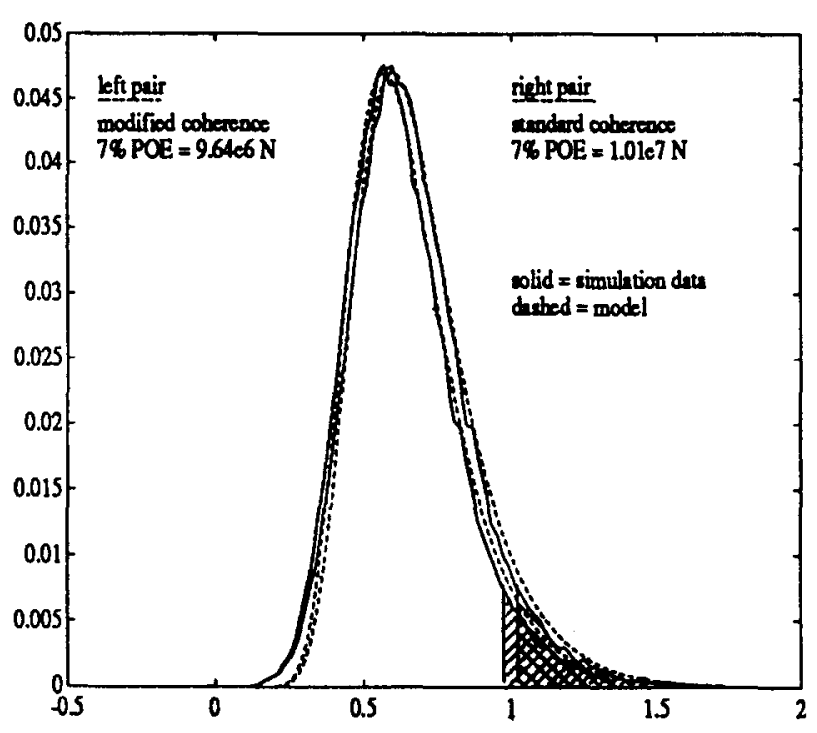

Fig. 15. Surge extreme wind loading $(\mathbf{N})$.

second moment statistics of the gust response factor are calculated after the simulation. The statistics of the tension in the windward tethers are also calculated as a linear transformation of the pitch response (i.e. pitch rotation times lever arm from structures centroid times axial tendon stiffness) and may be used as a preliminary bases for tether analysis.

The response is calculated in the surge, pitch, and yaw degrees of freedom, and is run separately for two different aerodynamic admittance function formulations, one using the standard coherence formulation and one using the modified coherence formulation given in this study for ocean-based structures. For both cases, aerodynamic and hydrodynamic damping effects are considered for surge response only. Figures 12-14 compare the admittance functions calculated using the

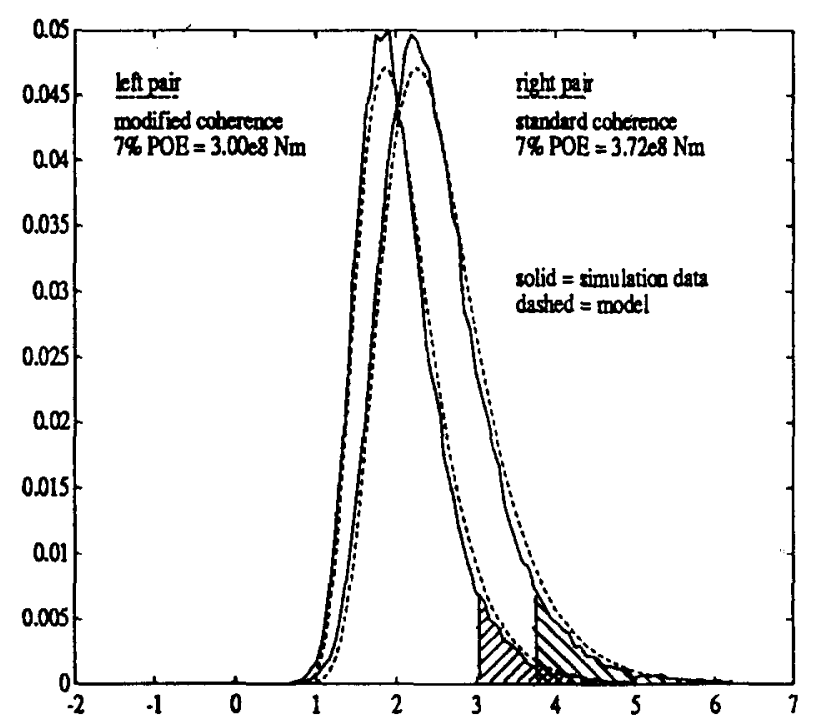

Fig. 16. Pitch extreme wind moment (Nm). 


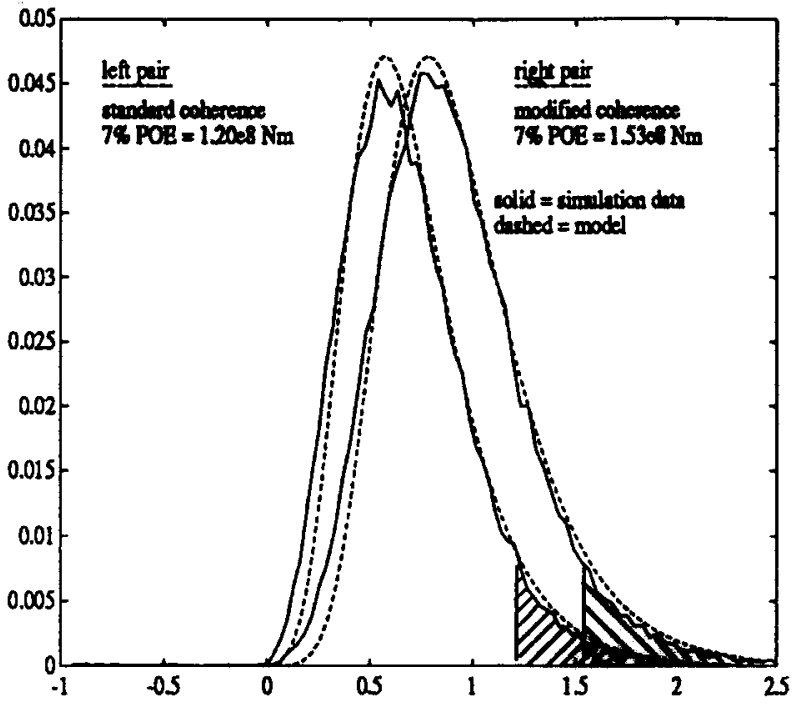

Fig. 17. Yaw extreme wind moment (Nm).

standard and modified coherence functions for surge, pitch and yaw motions, normalized to a unit maximum value. The admittance functions were calculated by adding the coherence over small TLP components (Fig. 11) in a double summation (eqns (27) and (28)). Figures 15-17 show the PDF of the surge, yaw and pitch extreme wind loading functions and Figs 18-20 are the PDF of the total TLP response calculated using the gust factor approach for the same three degrees of freedom. Figures 15-20 all show two pairs of plots; in each pair the solid line is the actual simulation data and the dashed line is a Type I Extreme Value distribution fitted to the data. The pairs represent the data applicable to the graph using the standard and modified coherence functions, with the 7\% POE identified in each pair.

The trends of the simulation results from standard to modified coherence formulations are presented in Tables 4-6 and agree well with the expected outcome predicted

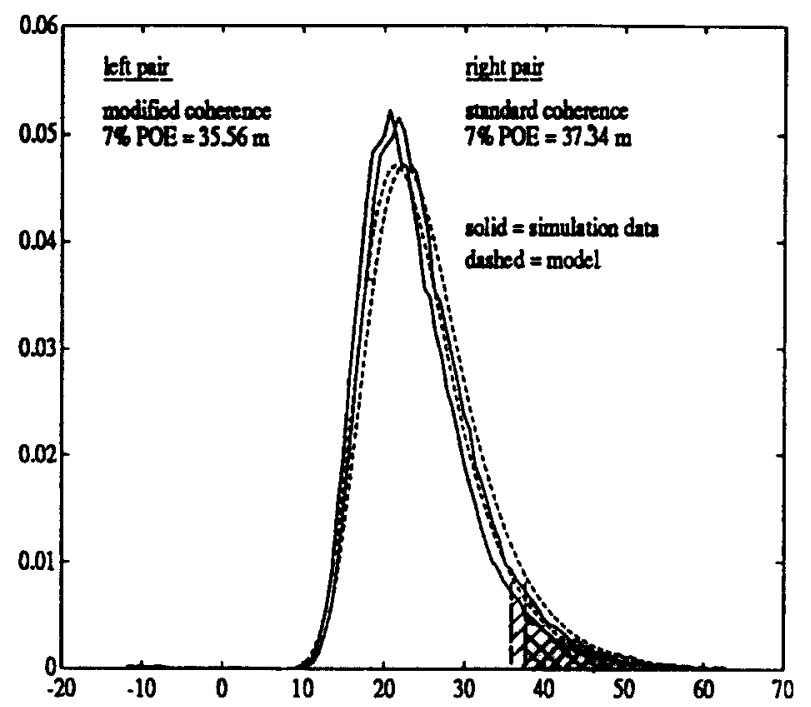

Fig. 18. Surge extreme response (m).

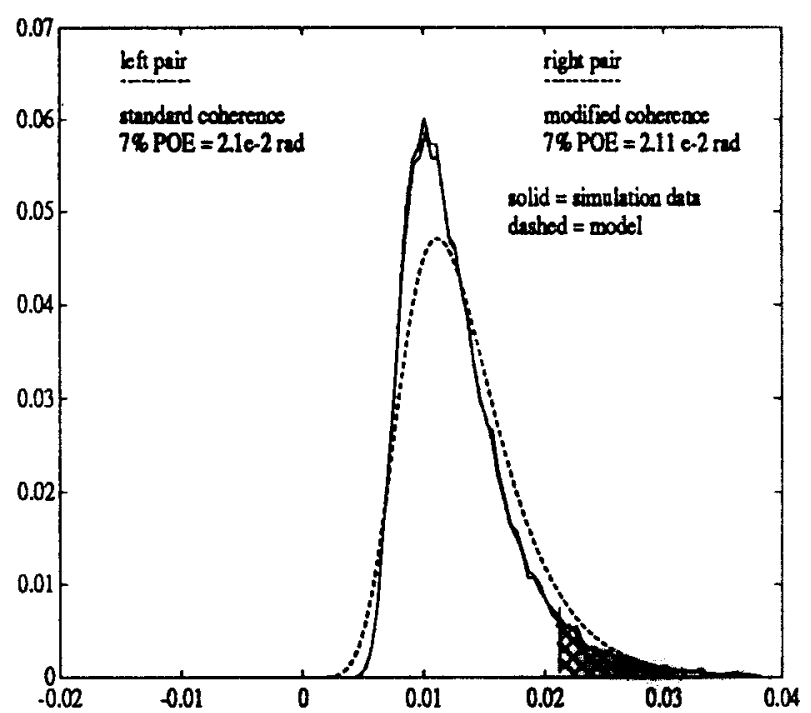

Fig. 19. Pitch extreme response (rad).

in Figs 12-14. The use of the modified conherence function in the admittance function lowers wind velocity correlation in the surge degree of freedom, which decreases rms response, and hence the gust factor. The modified admittance function in the pitch degree of freedom is slightly higher than the standard admittance function at the pitch natural frequency (Fig. 13), corresponding to a slightly higher dynamic pitch response and gust factor. The lower wind velocity correlation corresponding to the modified coherence function increases the yaw rms response and gust factor by increasing the level of unsymmetrical loading about the vertical axis. The mean response in all degrees of freedom remains the same for both cases since coherence is not a factor in the computation of static response.

The gust response factor calculated in this example may be used as a statistical measure of the most probable maximum response for future design consid-

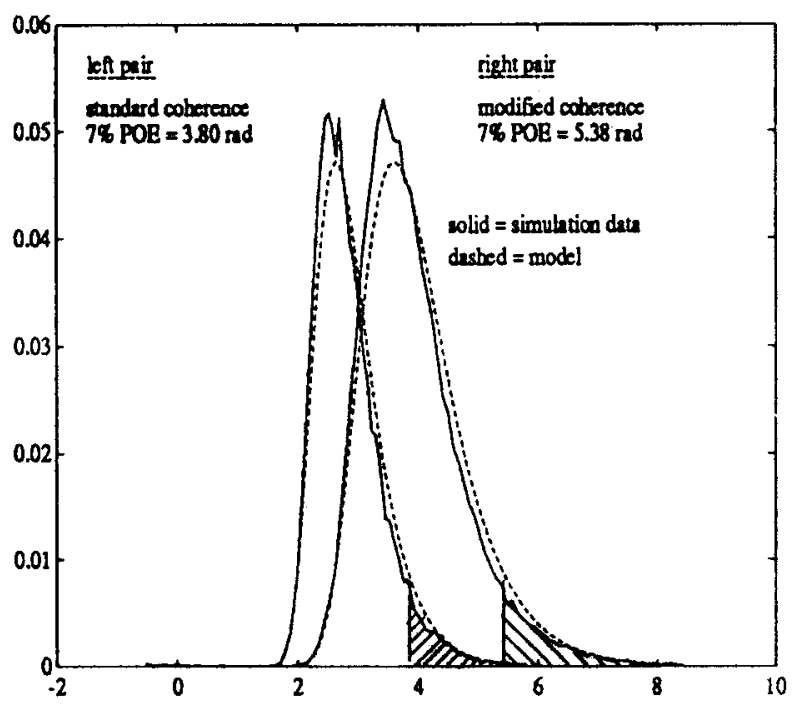

Fig. 20. Yaw extreme response (rad). 
Table 4. Surge response in TLP simulation

\begin{tabular}{|c|c|c|c|c|}
\hline \multirow[t]{2}{*}{ Surge response results } & \multicolumn{2}{|c|}{ Standard coherence } & \multicolumn{2}{|c|}{ Modified coherence } \\
\hline & Mean & $(\mathrm{COV})$ & Mean & $(\mathrm{COV})$ \\
\hline Static response & $0.1698 \mathrm{e} 2 \mathrm{~m}$ & $(0 \cdot 312)$ & $0.1698 \mathrm{e} 2 \mathrm{~m}$ & $(0 \cdot 312)$ \\
\hline Fluctuating response & $0.2951 \mathrm{e} 1 \mathrm{~m}$ & $(0 \cdot 283)$ & $0.2524 \mathrm{el} \mathrm{m}$ & $(0 \cdot 279)$ \\
\hline Total response & $0 \cdot 2546 \mathrm{e} 2 \mathrm{~m}$ & $(0 \cdot 293)$ & $0.2425 \mathrm{e} 2 \mathrm{~m}$ & $(0 \cdot 292)$ \\
\hline Gust factor & $1 \cdot 5052$ & $(0 \cdot 026)$ & $1 \cdot 433$ & $(0.024)$ \\
\hline Wind force & $0.6798 \mathrm{e} 7 \mathrm{~N}$ & $(0 \cdot 309)$ & 0.6470 e $7 \mathrm{~N}$ & $(0 \cdot 308)$ \\
\hline Wind force with POE of $7 \%$ & $0 \cdot 1015 \mathrm{e} 8 \mathrm{~N}$ & & 0.9645 e $7 \mathrm{~N}$ & \\
\hline
\end{tabular}

Table 5. Pitch response in TLP simulation

\begin{tabular}{|c|c|c|c|c|}
\hline \multirow[t]{2}{*}{ Pitch response results } & \multicolumn{2}{|c|}{ Stand coherence } & \multicolumn{2}{|c|}{ Modified coherence } \\
\hline & Mean & $(\mathrm{COV})$ & Mean & $(\mathrm{COV})$ \\
\hline Static response & $0.9000 \mathrm{e}-2 \mathrm{rad}$ & $(0 \cdot 311)$ & 0.9000 e- $2 \mathrm{rad}$ & $(0 \cdot 311)$ \\
\hline Fluctuating response & $0 \cdot 1033 \mathrm{e}-2 \mathrm{rad}$ & $(0.560)$ & $0 \cdot 1060$ e- $2 \mathrm{rad}$ & $(0.539)$ \\
\hline Total response & $0.131 \mathrm{e}-1 \mathrm{rad}$ & $(0.381)$ & $0.1321 \mathrm{e}-1 \mathrm{rad}$ & $(0 \cdot 377)$ \\
\hline Gust factor & $1 \cdot 432$ & $(0 \cdot 051)$ & $1 \cdot 445$ & $(0 \cdot 050)$ \\
\hline Static tendon tension & 0.1485 e $4 \mathrm{~N}$ & $(0 \cdot 311)$ & $0 \cdot 1485$ e $4 \mathrm{~N}$ & $(0 \cdot 311)$ \\
\hline Fluctuating tension & $0 \cdot 1701$ e3 & $(0.560)$ & 0.1750 e $3 \mathrm{~N}$ & $(0.539)$ \\
\hline Total tension & 0.216 e $4 \mathrm{~N}$ & $(0 \cdot 381)$ & $0 \cdot 2810$ e $4 \mathrm{~N}$ & $(0 \cdot 377)$ \\
\hline Tension gust factor & $1 \cdot 432$ & $(0.051)$ & $1 \cdot 445$ & $(0.050)$ \\
\hline Wint pitch moment & 0.2554 e9 $\mathrm{Nm}$ & $(0.287)$ & 0.2578 e $9 \mathrm{Nm}$ & $(0.258)$ \\
\hline Wind moment with POE of $7 \%$ & 0.3722 e9 $\mathrm{Nm}$ & & $0 \cdot 376039 \mathrm{Nm}$ & \\
\hline
\end{tabular}

Table 6. Yaw response in TLP simulation

\begin{tabular}{|c|c|c|c|c|}
\hline \multirow[t]{2}{*}{ Yaw response results } & \multicolumn{2}{|c|}{ Standard coherence } & \multicolumn{2}{|c|}{ Modified coherence } \\
\hline & Mean & $(\mathrm{COV})$ & Mean & $(\mathrm{COV})$ \\
\hline Static response & $0.4772 \mathrm{rad}$ & $(0 \cdot 815)$ & $0.4772 \mathrm{rad}$ & $(0 \cdot 721)$ \\
\hline Fluctuating response & $0.7917 \mathrm{rad}$ & $(0 \cdot 212)$ & $0 \cdot 1154$ el rad & $(0 \cdot 229)$ \\
\hline Total response & $0.2872 \mathrm{el} \mathrm{rad}$ & $(0 \cdot 203)$ & $0 \cdot 3958$ e $1 \mathrm{rad}$ & $(0 \cdot 226)$ \\
\hline Gust factor & $6 \cdot 3281$ & $(0 \cdot 216)$ & $8 \cdot 5523$ & $(0 \cdot 146)$ \\
\hline Wind yaw moment & $0.6957 \mathrm{e} 8 \mathrm{Nm}$ & $(0 \cdot 453)$ & $0.9301 \mathrm{e} 8 \mathrm{Nm}$ & $(0 \cdot 403)$ \\
\hline Wind force with POE of $7 \%$ & $0.1199 \mathrm{e} 9 \mathrm{Nm}$ & & 0.1528 e $9 \mathrm{Nm}$ & \\
\hline
\end{tabular}

Table 7. Reliability analysis of TLP surge response

\begin{tabular}{|c|c|c|c|c|c|}
\hline \multirow{2}{*}{$\begin{array}{l}\text { Normal } \\
\text { distribution } \\
(\mathrm{COV}=0 \cdot 20) \\
\text { Mean tolerance }\end{array}$} & \multirow{2}{*}{$\begin{array}{c}\text { Extreme } \\
\text { distribution } \\
(\mathrm{COV}=0 \cdot 293) \\
\text { Mean response }\end{array}$} & \multicolumn{4}{|c|}{ Probability of failure obtained by different methods } \\
\hline & & FORM & SORM & $\begin{array}{l}\text { Directional } \\
\text { simulation } \\
\quad(\mathrm{rms})\end{array}$ & $\begin{array}{c}\text { MC } \\
\text { simulation } \\
\text { (rms) }\end{array}$ \\
\hline $35 \mathrm{~m}$ & $25 \cdot 46 \mathrm{~m}$ & $0 \cdot 1516$ & $0 \cdot 1658$ & $\begin{array}{c}0 \cdot 1658 \\
(3.85 \mathrm{e}-4)\end{array}$ & $\begin{array}{l}0 \cdot 1706 \\
(2 \cdot 66-3)\end{array}$ \\
\hline $37 \mathrm{~m}$ & $25 \cdot 46 \mathrm{~m}$ & $0 \cdot 1193$ & $0 \cdot 1318$ & $\begin{array}{c}0.1312 \\
(1.85 \mathrm{e}-4)\end{array}$ & $\begin{array}{c}0.1295 \\
(2.37 \mathrm{e}-3)\end{array}$ \\
\hline $39 \mathrm{~m}$ & $25 \cdot 46 \mathrm{~m}$ & 0.09403 & $0 \cdot 1047$ & $\begin{array}{c}0.1046 \\
(6.62 \mathrm{e}-5)\end{array}$ & $\begin{array}{c}0 \cdot 1042 \\
(2 \cdot 16 \mathrm{e}-3)\end{array}$ \\
\hline $40 \mathrm{~m}$ & $25 \cdot 46 \mathrm{~m}$ & 0.08347 & 0.09342 & $\begin{array}{c}0.09337 \\
(4.52 \mathrm{e}-5)\end{array}$ & $\begin{array}{c}0.0950 \\
(2.07 \mathrm{e}-3)\end{array}$ \\
\hline
\end{tabular}

erations of this TLP. On its simplest level this analysis could be done by approximating the TLP as a fully correlated structure and using equivalent static loading based on a gust loading factor of the wind velocity to determine a maximum response. The advantage of the approach used here is the inclusion of the fluctuating response as well as the calculation of statistical uncertainty in all response descriptions. The results of the above example may now be used as a basis for reliability analysis.

The statistics of the total surge response in the above simulation are used as the loading function $S$ in a reliability analysis. The goal of this analysis is to determine the probability of surge response exceeding 
a predefined acceptable limit. The first and second moments of total response are used to define an extreme value distribution for the load term in the limit state, and the resistance is represented as a normallydistributed displacement tolerance level, based on the mean static surge response of the above simulation. The mean tolerance is varied over a range of values to determine the sensitivity of the probability of failure to the tolerance. Table 7 lists the input and results of this analysis. It is observed that the SORM, Directional simulation, and Monte Carlo simulation methods give very similar predictions for the probability of failure, while the Form predicts a lower failure probability. It is not always the case that SORM will predict a higher failure probability than FORM, this is dependent on whether the second-order approximation of the limit state is concave or convex. However, because of the nonlinear nature of the failure surface, and the nonnormal distribution of the load function, the results of the FORM tend to be often the least reliable.

The Directional and MC simulations used approximately the same CPU time, but the Directional simulation results in a significantly lower standard deviation from the calculated probability of failure. It is important to note that the seemingly good comparison between MC and Directional simulation has much to do with both the simplicity of the limit state used in this example, and the relatively high probability of failure. For applications using more nonlinear limit states involving non-normal PDFs, Directional simulation becomes more attractive since the Monte Carlo simulation would require significantly more sampling and CPU time to match its accuracy. As the probability of failure gets smaller the computer time required by a straightforward MC simulation to yield statistically meaningful results increases, thus rendering importance sampling simulation techniques more appropriate.

A more detailed reliability analysis for various modes of failure could be performed along these same lines. The purpose here is simply to demonstrate the applicability of the gust factor approach to reliability analysis.

\section{CONCLUDING REMARKS}

The gust loading factor approach is formulated for offshore applications with particular reference to TLPs. This technique permits quantification of both the most probable extreme loads in terms of equivalent static loading that accounts for unsteadiness in wind, and amplification in the load effects due to structural sensitivity to wind-induced response. Single-point wind statistics are used as the basis for characterizing the wind field at a point. Wind loading on small structures may be assumed to be fully correlated and an equivalent static analysis based on a single-point wind field representation may be appropriate. However, multipoint representation of the wind field is necessary for the dynamic analysis of large offshore structures where eddies in the wind field may not encompass the entire structure. The energy content of wind over the ocean differs from that of wind over land at low frequencies, and horizontally compliant structures are likely to experience dynamic amplification in this frequency range. A spectral model of ocean wind is examined to accurately assess the wind loading experienced by offshore structures. Partial wind correlation over the structure is modeled using a modified coherence function developed specifically for structures subjected to ocean wind loading environments. The most probable extreme deviation from mean wind speed is statistically determined using the gust loading factor approach and used to determine the loading of an example TLP offshore platform. The presence of waves and currents is represented in terms of equivalent damping ratios. The gust factor approach is extended to predict extreme response statistics of a $T L P$ which are then applied to a reliability analysis based on an acceptable magnitude of surge response.

\section{ACKNOWLEDGMENT}

Support for this study provided in part by NSF grant BCS-9096274 and NSF Engineering Research Centers program grant CDR-871512. Any opinions, findings, or recommendations expressed in the paper are those of the author's and do not necessarily represent the views of the sponsors.

\section{REFERENCES}

1. Kareem, A., Dynamic Effects of Wind on Offshore Structures. Offshore Technology Conference, OTC 3764 1980.

2. Kareem, A. \& Dalton, C., Dynamic Effects of Wind on Tension Leg Platforms. Proceedings, Ocean Structural Dynamics Symposium '82, Oregon State University, Corvallis, Sept. 1982. pp. 563-85.

3. Salvesen, N., Von Kerczek, C.H., Yue, D.K. \& Stern, F., Computation of Nonlinear Surge Motion of Tension Leg Platforms. Offshore Technology Conference, OTC 4394, Houston, 1982.

4. Simiu, E. \& Leigh, S.D., Turbulent Wind and Tension Leg Platform Surge. J. Struct. Eng., 110 (1984) 785-802.

5. Kareem, A., Wind-Induced Response Analysis of Tension Leg Platforms. Journal of the Structural Division (ASCE), $111(1)(1985) 37-55$.

6. Vickery, P., Wind and Wave Loads on a Tension Leg Platform: Theory and Experiment. Dissertation, University of Western Ontario, 1988.

7. Vickery, B.J., Wind Loads on Compliant Offshore Structures. Proceedings, Ocean Structural Dynamics Symposium '82, Oregon State University, Corvallis, Sept. 1982. pp. $632-46$. 
8. Davenport, A.G., Gust Loading Factors. Journal of the Structural Division ( $A S C E$ ) 93(ST3) (1976).

9. Simiu, E. \& Scanlan, Wind Effects on Structures: An Introduction to Wind Engineering. John Wiley and Sons, New York, 1985.

10. Large, W.G. \& Pond, C., Open Ocean Momentum Flux Measurements in Modern to Strong Winds. J. Phys. Ocean 11 (1981) pp. 1103-105.

11. ESDU, Strong Winds in the Atmospheric Boundary Layer, Part I: Mean Hourly Wind Speeds. Engineering Science Data Unit, London, 1982, 82026.

12. Forristall, G.I., Wind Spectra and Gust Factors Over Water. Proc. of the 20th Annual Offshore Technology Conference, OTC 5735, Houston, 1988, pp. 449-60.

13. Ochi, M.K. \& Shin, Y.S., Wind Turbulent Spectra for Design Consideration of Offshore Structures. Proc. of the 20th Annual Offshore Technology Conference, OTC 5736, Houston, 1988, pp. 461-7.

14. Harris, R.I., Some Further Thoughts on the Spectrum of Gustiness in Strong Winds. J. Wind Engng. Ind. Aerod. 33 (1990) 461-77.

15. Kareem, A., Wind Effects on Structures: A Probabilistic Viewpoint. Probabilisitc Engineering Mechanics 2(4) (1987) 166-200.

16. Kristensen, L. \& Jensen, N.O., Lateral Coherence in Isotropic Turbulence and in the Natural Wind. Boundary Layer Meteorology 17 (1979) 353-73.

17. Vickery, B.J. \& Pike, P.J., An Investigation of Dynamic Wind Loads on Offshore Platforms. Proceedings Offshore Technology Conference, OTC 4955, Houston, 1985.

18. ESDU, Characteristics of Atmospheric Turbulence Near the Ground, Part II: Single Point Data for Strong Winds (Neutral Atmosphere). Engineering Sciences Data Unit, Data Item Number 85020, London, 1985.

19. Li, Y. \& Kareem, A., ARMA systems in Wind Engineering. Probabilistic Engineering Mechanics, 5(2) (1990) 50-9.

20. Reed, D.A. \& Scanlan, R.H., Autoregressive Representation of Longitudinal Lateral, and Vertical Turbulence Spectra. J. Wind Engng Ind. Aerod., 17 (1984) 199-214.

21. Samaras, E., Shinozuka, M. \& Tsurui, A., ARMA Representation of Random Process. Journal of Engineering Mechanics, 111 (1984) 449-61.

22. Spanos, P.D. \& Mignolet, M.P., MA to ARMA Modeling of Wind. Proceedings of the 6th U.S. National Conference on Wind Engineering, ed. A. Kareem, 1989.

23. Kareem, A. \& Li, Y., Stochastic Response of a Tension Leg Platform to Wind and Wave Fields. Department of Civil Engineering, University of Houston Technical report No. UHCE 88-18, 1988

24. Kareem, A., Nonlinear Wind Velocity Term and Response of Compliant Offshore Structures. Journal of Engineering Mechanics (ASCE), 110 (1984) 1573-8.

25. Davenport, A.G., The Spectrum of Horizontal Gustiness Near the Ground in High Winds. Q.J. Roy. Met, Soc., 87 (1961) 194-211.

26. Harris, R.I., The Nature of Wind, Proc. of the Modern Design of Wind Sensitive Structures, Construction. Industry Research and Information Association, London, UK, 1971.

27. Greenway, M.E., The Effects of Finite Observation Time and Finite Averaging Time on the r.m.s. and Extreme Wind Velocity. J. Ind. Aerod. 6 (1980) 175-9.

28. Rice, S.O., Mathematical Analysis of Random Noise. Bell Syst. Tech. J., 23 (1944), 282-332; 24 (1945) 45-156.

29. Gumbel, E.J., Statistics of Extremes. Columbia University Press, New York, 1958.

30. Zhao, J. \& Kareem, A., Response Statistics of Tension Leg Platforms Under Wind Loads. Proceedings of ASCE
Speciality Conference, Civil Engineering in Oceans, College Station, TX, 1992.

31. Moe, G. \& Verley, R.L.P., Hydrodynamic Damping of Offshore Structures in Waves and Currents. Proceedings Offshore Technology Conference, OTC 3789, 1980.

32. Bearman, et al. The Loading on a Cylinder in Post-Critical Flow Beneath Periodic and Random Waves. Behavior of Offshore Structures. Elsevier Science Publishers B.V., Amsterdam, 1985.

33. Sarpkaya, T., Forces on a Circular Cylinder in Viscous Oscillatory Flow at Low Keulegan-Carpenter Numbers. Journal of Fluid Mechanics, 165 (1986) 61-71.

34. Marthinsen, T., Hydrodynamics in TLP Design. Eighth International Conference on Offshore Mechanics and Arctic Engineering, The Hague, 19-23 March 1989.

35. Batchelor, G.K. An Introduction to Fluid Dynamics. Cambridge University Press, 1970

36. Eatock Taylor, R. \& Rajagopalan, A., Load Spectra for Slender Offshore Structure in Waves and Currents. Earthquake Engineering and Structural Dynamics, 11 (1983) 831-42.

37. Kareem, A. \& Li, Y., Wind Excited Surge Response of a Tension Leg Platform: A Frequency Domain Approach. Journal of Engineering Mechanics, (ASCE), 119(1) 1993.

38. PROBAN - Computer Program for Probabilistic Reliability and Sensitivity Analysis. Det Norske Veritas, Veritas Sesam Systems A.S., Hovik, Norway, 1989.

39. Cartwright, D.E. \& Longuet-Higgins, M.S., The Statistical Distribution of Maxima of a Random Function. Proc. Royal Society, 237 (1957).

40. Kristensen, L., Casonova, M., Courtney, M.S. \& Troen, I., In Search of a Gust Definition. Boundary Layer meterology, 55 (1991) 91- 107.

41. Robson, J.D., An Introduction to Random Vibration. Elsevier Publishing Company, New York, 1964.

42. Davenport, A.G., Note on the Distribution of the largest Value of a Random Function with Application to Gust Loading. Proceedings of the Institution of Civil Engineers 28 (1964) 187-98.

43. Preumont, A., On the Peak Factor of Stationary Gaussian Processes. J. of Sound and Vibration, 100 (1) (1985) 15-34.

\section{APPENDIX: METHODS OF EXTREME EXCURSION ANALYSIS}

Two widely-used methods of excursion analysis are the Rice approach (1944) and the Gumbel approach $(1958){ }^{28,29,39}$ Both consider a stationary time series $u(t)$ with zero mean divided into an ensemble of $M$ realizations.

Rice considers the number of excursions beyond some level $U$ represented as $N_{i}(U)$ where $i$ denotes the $i$ th of the $M$ realizations. Averaging over the ensemble gives the mean number of excursions beyond $U$ for the proces $u(t)$.

$$
N(U)=\lim _{M \rightarrow \infty} \frac{1}{M} \sum_{i=1}^{M} N_{i}(U)
$$

Assuming the individual excursions beyond $U$ are statistically independent in each realization, the excursions are Poisson distributed as rare events. The expression for the probability of $n$ excursions beyond 
$U$ is then

$$
P_{U}(n)=\frac{\mathrm{e}^{-N(U)}}{n !} N^{n}(U)
$$

In order to validate the assumption of statistical independence, the probability of zero excursions is considered $^{40}$

$$
P_{U}[0]=\mathrm{e}^{-N(U)}
$$

Expressing the exceedence value $U$ as some value $q$ times the rms value of the process $\sigma_{u}$, the expected number of excursions beyond $q \sigma_{u}$ is commonly accepted as ${ }^{41,42}$

$$
E(q)=\nu \mathrm{e}^{\left(-q^{2} / 2\right)}
$$

Where $E(q)$ is the expected number of excursions beyond $q \sigma_{u}$ per unit time $T$ and is related to the average excursion throughout the ensemble by $N(U)=T E(q)$. The cyclic rate $\nu$ may be expressed as

$$
\nu=\frac{\sigma_{u}}{\sigma_{u}}=\left[\frac{\int_{0}^{\infty} f^{2} S_{u}(f) \mathrm{d} f}{\int_{0}^{\infty} S_{u}(f) \mathrm{d} f}\right]^{1 / 2}
$$

where $S_{u}$ is the PSD of the process $u(t)$. The cyclic rate physically is the frequency at which most of the energy in the spectrum is concentrated; for lightly damped systems this may be approximated as the natural frequency of the system. ${ }^{42}$

The probability of zero excursions beyond $U$ in eqn (A3) may be viewed as the probability that the ratio $Q$ of the largest peak to $\sigma_{u}$ is less than $q$ in the interval $T$. Based on eqn (A3) the corresponding probability density of $Q$ for time interval $T$ is given by

$$
P_{Q}(q)=T E(q) \mathrm{e}^{-E(q) T}
$$

The expectation of the largest value $Q$ in the interval $T$ is then

$$
E(Q)=\int_{-\infty}^{\infty} q P_{Q}(q) \mathrm{d} q=\int_{-\infty}^{\infty} q T E(q) \mathrm{e}^{-E(q) T} \mathrm{~d} q
$$

This is known as the peak factor and was evaluated by Davenport ${ }^{42}$ as approximately

$$
E(Q)=(2 \ln (\nu T))^{1 / 2}+\frac{0 \cdot 5772}{(2 \ln (\nu T))^{1 / 2}}
$$

with a corresponding standard deviation expressed as

$$
\sigma_{Q}=(\Pi / \sqrt{6}) /(2 \ln (\nu T))^{1 / 2}
$$

The peak factor $E(Q)$ is usually represented by $g$. This convention is used in the main body of this paper.

A modification of this formulation considers the bandwidth of the process and is based on the
Table A1. Comparison of peak factor statistics

\begin{tabular}{lccc}
\hline & $\begin{array}{l}\xi=0.05,0 \cdot 15 \\
(\mathrm{~A} 8)(\mathrm{A} 9)\end{array}$ & $\begin{array}{c}\xi=0.05 \\
(\mathrm{~A} 10)(\mathrm{A} 11)\end{array}$ & $\begin{array}{c}\xi=0 \cdot 15 \\
(\mathrm{~A} 10)(\mathrm{A} 11)\end{array}$ \\
\hline Peak factor & 2.884 & 2.673 & 2.770 \\
rms & 0.4790 & 0.5266 & 0.4217 \\
\hline
\end{tabular}

assumption that the extreme point process is Markovian. This leads to the expressions ${ }^{43}$

$$
E(Q)=\left(2 \ln \left(\kappa_{u} \nu t\right)\right)^{1 / 2}+\frac{0.5772}{\left.2 \ln \left(\kappa_{u} \nu T\right)\right)^{1 / 2}}
$$

and

$$
\sigma_{Q}=(\Pi / \sqrt{6}) /\left(2 \ln \left(\kappa_{\alpha} \nu T\right)\right)^{1 / 2}
$$

where $\kappa_{\alpha}$ and $\kappa_{u}$ are functions of the process bandwidth only. For processes with a wide bandwidth $(\xi>0 \cdot 1)$ the peak factor becomes essentially independent of the bandwidth, as is the case for fluctuating winds. Table Al compares the peak response factor for processes with bandwidths of 0.05 and $0 \cdot 15$. The sampling time $T$ is one hour, and the cyclic rate is the natural frequency of the TLP surge response $(0.01 \mathrm{~Hz})$.

The Gumbel approach is to record the maximum value $U_{i}$ in $u(t)$ in each of the $M$ realizations. The probability of not exceeding $q \sigma_{u}$ is written as

$$
P\left(u<q \sigma_{u}\right)=\lim _{M \rightarrow \infty} \frac{1}{M} \sum_{i=1}^{M} R_{i}\left(q \sigma_{u}\right)
$$

where

$$
R_{i}=\left(\begin{array}{ccc}
1 & \text { for } & U_{\max } \leq a \sigma_{u} \\
0 & \text { for } & U_{\max }>q \sigma_{u}
\end{array}\right)
$$

$P\left(u<q \sigma_{u}\right)$ is approximated by a Tyle I Extreme Value cumulative distribution function developed by Gumbel $^{29}$

$$
P\left(u<q \sigma_{u}\right)=\mathrm{e}\left(-\mathrm{e}\left(-\alpha_{n}\left(x-u_{n}\right)\right)\right)
$$

where $\alpha_{n}$ and $u_{n}$ are the scale and location parameters, respectively. They are defined with respect to the mean and rms of the extreme signal such that mean $=u_{n}+0.5772 / \alpha_{n}$ and $\mathrm{rms}=\pi /\left(\sqrt{6} \alpha_{n}\right)$.

For the Rice approach, the probability that $U$ is not exceeded is given by eqn (A3). If $U$ is not exceeded, the maximum value will not exceed $U$ either. Similarly, the Gumbel approach finds the probability that the maximum value of $u(t)$ does not exceed $U$ in its duration $P\left(u<q \sigma_{u}\right)$. It is observed that both approaches are based on finding the same probability

$$
P\left(u<q \sigma_{u}\right)=P_{U}[0]
$$

where $U=q \sigma_{u}$, and may be considered equivalent. This equivalence was also cited by Davenport. ${ }^{42}$ Both approaches lead to the Type I Extreme Value distribution as a reasonably accurate extreme value description. 